\title{
Thermodynamic Equilibrium as a Symmetry of the Schwinger-Keldysh Action
}

\author{
L. M. Sieberer ${ }^{1,2}$, A. Chiocchetta ${ }^{3}$, A. Gambassi ${ }^{3}$, U. C. Täuber ${ }^{4}$, and S. Diehl ${ }^{5}$ \\ ${ }^{1}$ Department of Condensed Matter Physics, Weizmann Institute of Science, Rehovot 7610001, Israel \\ ${ }^{2}$ Institute for Theoretical Physics, University of Innsbruck, A-6020 Innsbruck, Austria \\ ${ }^{3}$ SISSA - International School for Advanced Studies and INFN, via Bonomea 265, I-34136 Trieste, Italy \\ ${ }^{4}$ Department of Physics (MC 0435), Robeson Hall, 850 West Campus Drive, Virginia Tech, Blacksburg, Virginia 24061, USA and \\ ${ }^{5}$ Institute of Theoretical Physics, TU Dresden, D-01062 Dresden, Germany
}

(Dated: November 11, 2015)

\begin{abstract}
Extended quantum systems can be theoretically described in terms of the Schwinger-Keldysh functional integral formalism, whose action conveniently describes both dynamical and static properties. We show here that in thermal equilibrium, defined by the validity of fluctuation-dissipation relations, the action of a quantum system is invariant under a certain symmetry transformation and thus it is distinguished from generic systems. In turn, the fluctuation-dissipation relations can be derived as the Ward-Takahashi identities associated with this symmetry. Accordingly, the latter provides an efficient test for the onset of thermodynamic equilibrium and it makes checking the validity of fluctuation-dissipation relations unnecessary. In the classical limit, this symmetry reduces to the well-known one which characterizes equilibrium in the stochastic dynamics of classical systems coupled to thermal baths, described by Langevin equations.
\end{abstract}

PACS numbers: 05.30.Jp,05.40.-a,05.70.Ln

\section{INTRODUCTION}

In recent years, the question under which conditions and how a quantum many-body system thermalizes has received ever-growing attention. This interest has been primarily triggered by the increasing ability to prepare and manipulate such systems, which might be either isolated [1-3] — as it is typically the case in experiments with cold atoms [4, 5] — or in contact with an environment (open), and therefore subject to losses and driving.

After an abrupt perturbation, isolated systems are generically expected to thermalize in the sense that expectation values of local quantities at long times can be determined on the basis of suitable statistical ensembles $[1,3]$. However, this might not be the case because of the presence of an extensive amount of conserved quantities induced by integrability [6-10] or because of a breaking of ergodicity due to the occurrence of many-body localization [11-14]. Although it is possible to define a variety of effective temperatures based on the static $[15,16]$ and dynamic properties $[17,18]$ under such circumstances, the lack of thermal behavior is witnessed by the fact that these temperatures do not necessarily take all the same thermodynamic value.

Examples of open systems include exciton-polaritons in semiconductor heterostructures $[19,20]$, arrays of microcavities [21, 22], trapped ions [23], as well as optomechanical setups [24]. In general it is unclear, a priori, by which physical mechanism an effective temperature is possibly established in these systems and, in case, what determines its value. Recent work, however, suggests possible mechanisms where an effective temperature can occur as a consequence of the competition between driven-dissipative and coherent dynamics [2533]. Irrespective of its cause, effective thermalization often affects only the low-energy degrees of freedom [16, 25-37].

All these examples show clearly that the presence of effective thermodynamic equilibrium (which might be established only in a subsystem or within a specific range of frequencies) in the steady state of a system is often by no means obvious. Hence, before addressing the question of whether the time evolution of a certain system leads to thermalization or not, it is imperative to identify criteria which allow a clearcut detection of thermodynamic equilibrium conditions in the stationary state. In this regard, it is important to consider not only the static properties of the density matrix of the system, which describes its stationary state, but also the dynamics of fluctuations: being encoded, e.g., in two-time correlation and response functions, it might or might not be compatible with equilibrium. As a fundamental difference between static and dynamic properties, the latter necessarily involves the generator of time evolution, while the former does not.

In this work we consider the following operative definition of thermal equilibrium: a system is in thermal equilibrium at a certain temperature $T$ if expectation values of arbitrary products of operators, evaluated at different times, are connected by quantum fluctuation-dissipation relations (FDRs) involving the temperature $T$. These FDRs were shown [38-40] to be equivalent to a combination of the quantum-mechanical timereversal transformation [41] and the Kubo-Martin-Schwinger (KMS) condition [42, 43]. Heuristically, the latter condition expresses the fact that the Hamiltonian ruling the time evolution of a system is the same as that one determining the density matrix of the canonical ensemble, which characterizes the system when it is weakly coupled to a thermal bath. In both the generalized FDRs and the KMS condition the temperature appears as a parameter.

From the theoretical point of view, static and dynamical properties of statistical systems (both classical and quantum) are often conveniently studied in terms of dynamical functionals, which are used in order to generate expectation values of physical observables in the form of functional integrals over a suitable set of fields. Then, it is natural to address the issue of the possible equilibrium character of the stationary state by investigating the properties of the corresponding dynamical functional. In the case of classical statistical systems evolving 
under the effect of an external stochastic noise of thermal origin, this issue has been discussed to a certain level of detail in the past [44-49], and it was found that the dynamical functional acquires a specific symmetry in thermodynamic equilibrium. As in the case of the FDRs and the KMS condition, the (inverse) temperature $\beta=1 / T$ enters as a parameter in this symmetry transformation. Remarkably, classical FDRs can be derived as a consequence of this symmetry. For quantum systems, instead, we are not aware of any analogous derivation based on the symmetries of the corresponding dynamical functional, which takes the form of a Schwinger-Keldysh action (see, e.g., Refs. 50-57).

The aim of the present work is to fill in this gap by showing that also the Schwinger-Keldysh dynamical functional of a quantum system in thermal equilibrium is characterized by a specific symmetry, i.e., it is invariant under a certain transformation $\mathcal{T}_{\beta}$. This symmetry may be considered as the generalization of the classical one mentioned above, to which it reduces in a suitable classical limit [58]. In addition, $\mathcal{T}_{\beta}$ can be written as a composition of the quantum-mechanical time reversal expressed within the Schwinger-Keldysh formalism — reflecting a property of the generator of dynamics - and of the transformation which implements the KMS conditions, associated with a property of the state in question. The existence of this symmetry was already noticed in Ref. 58 for mesoscopic quantum devices, where it was used to derive fluctuation relations for particle transport across them. However, to our knowledge, the connection between this symmetry and the presence of equilibrium conditions has not yet been established.

The rest of the presentation is organized as follows: the key results of this work are anticipated and summarized in Sec. II; in Sec. III, we specify the symmetry transformation $\mathcal{T}_{\beta}$, provide its various representations, and list a number of properties which are then detailed in Sec. IV. In particular, we discuss the invariance of unitary time evolution in Sec. IV A, while in Sec. IV B we consider possible dissipative terms which are invariant under $\mathcal{T}_{\beta}$. We discuss how the quantum symmetry reduces in the limit $\hbar \rightarrow 0$ to the one known in classical stochastic systems in Sec. IV C. As we discuss in Sec. V, the symmetry can be interpreted as a practical implementation of the KMS condition on the Schwinger-Keldysh functional integral. Finally, Sec. VI presents applications of the equilibrium symmetry: in Sec. VI A we derive the FDR for two-point functions while in Sec. VI B we show that the steady states of a quantum master equation explicitly violate the symmetry. The case of a system driven out of equilibrium by a coupling with two baths at different temperature and chemical potential is considered in Sec. VIC; Sec. VID briefly touches upon a number of other applications of the symmetry.

\section{KEY RESULTS}

a. The invariance under $\mathcal{T}_{\beta}$ of the Schwinger-Keldysh action is a sufficient and necessary condition for a system to be in thermal equilibrium. As mentioned in Sec. I, we consider a system to be in thermal equilibrium if all the FDRs are satis- fied with the same temperature $T=\beta^{-1}$ or, equivalently [38$40]$, if the KMS condition (combined with time reversal) is satisfied. In Sec. V, we show that these conditions imply the thermal symmetry $\mathcal{T}_{\beta}$ of the Schwinger-Keldysh action corresponding to the stationary state of the system. Conversely, the fluctuation-dissipation relations can be derived as consequences of the symmetry, proving their equivalence.

b. A different perspective: thermal equilibrium as a symmetry. A key conceptual step forward we take in this work is to provide a compact formulation of thermal equilibrium conditions of a quantum system - i.e., the KMS condition (or, alternatively, of the equivalent hierarchy of FDRs) in terms of a single symmetry $\mathcal{T}_{\beta}$, which can be considered as the fundamental property of quantum systems in thermal equilibrium. This perspective is especially fruitful within the field-theoretical formalism, where various tools have been developed to work out the consequences of the symmetries of the action of a given system. In this context, for example, the hierarchy of generalized quantum FDRs can be derived straightforwardly as the Ward-Takahashi identities associated with the thermal symmetry (see Secs. V and VIA). In addition, the Schwinger-Keldysh formalism provides a convenient framework to take advantage of very powerful and efficient renormalization-group techniques for studying the possible emergence of collective behaviors and for monitoring how the effective description of a statistical system depends on the length and time scale at which it is analyzed. The possible scale dependence of the restoration/violation of the equilibrium symmetry could shed light on the mechanism underlying the thermalization of extended systems.

As we mentioned above, the idea of viewing thermal equilibrium as a symmetry is certainly not new. However, while previous studies were primarily concerned with classical statistical physics [44-49], here we generalize this idea to the quantum case.

c. Unification of the quantum and classical description of equilibrium systems. As pointed out in Ref. 58, the equilibrium symmetry reduces, in the classical limit, to a known symmetry which characterizes thermal equilibrium in open classical systems [44-49]. In Sec. IV C we review the classical limit of the Schwinger-Keldysh action for a system coupled to a thermal bath $[55,56]$ and we discuss in detail how the classical equilibrium symmetry is recovered. The comparison with the classical symmetry highlights some remarkable differences with the quantum case: in fact, in classical systems, thermal equilibrium can be regarded as a consequence of detailed balance, which, in turn, is related to the property of microreversibility of the underlying microscopic dynamics. In fact, the classical equilibrium symmetry is derived by requiring the dynamical functional to satisfy these properties [4648]. For quantum system, instead, an analogous satisfactory definition of detailed balance and microreversibility is seemingly still missing, leaving open the important question about the very nature of thermal equilibrium of quantum systems.

d. Efficient check for the presence of thermodynamic equilibrium conditions. The symmetry is of great practical value, as it reduces answering the question about the possible presence of thermodynamic equilibrium to verifying a symmetry 
of the Schwinger-Keldysh action instead of having to check explicitly the validity of all FDRs. In particular, we show in Sec. VIB that the Markovian quantum dynamics described by a Lindblad master equation $[59,60]$ explicitly violates the symmetry. This reflects the driven nature of the system: indeed, the Lindblad equation may be viewed as resulting from the coarse graining of the evolution of an underlying timedependent system-bath Hamiltonian, with a time dependence dictated by coherent external driving fields.

Moreover, in Sec. VIC we consider a bosonic mode coupled to two baths at different temperatures and chemical potentials: in this case, the resulting net fluxes of energy or particles drive the system out of equilibrium with a consequent violation of the symmetry.

e. A new perspective on the construction of the Schwinger-Keldysh action. At the conceptual level, the existence of the symmetry provides a new perspective on the construction of Schwinger-Keldysh functional integrals. In particular, as customary in quantum field theories, one may consider the symmetry as the fundamental principle: indeed, it is explicitly present for any time-independent (time-translation invariant) Hamiltonian which generates the dynamics of a system at the microscopic scale. Then, requiring the symmetry to hold for the full effective Keldysh action at a different scale fixes the admissible dissipative terms so as to satisfy FDRs between response and correlation functions of arbitrary order; translating back into the operator language, this provides a concrete hint why stationary density matrices of the form $\rho \sim e^{-\beta H}$ are favored over arbitrary functions $\rho(H)$ for the description of static correlation functions.

\section{SYMMETRY TRANSFORMATION}

As we anticipated above, a convenient framework for the theoretical description of the time evolution of interacting quantum many-body systems is provided by the SchwingerKeldysh functional integral formalism [55, 56]. It offers full flexibility in describing both non-equilibrium dynamics and equilibrium as well as non-equilibrium stationary states, which is out of reach of the finite-temperature Matsubara technique [61]. In addition, it is amenable to the well-established toolbox of quantum field theory. The simplest way to illustrate the basic ingredients of the Schwinger-Keldysh formalism is to consider the functional integral representation of the so-called Schwinger-Keldysh partition function $Z$. For a system with unitary dynamics generated by the Hamiltonian $H$ and initialized in a state described by a density matrix $\rho_{0}$, this function is given by $Z=\operatorname{tr}\left(e^{-i H t} \rho_{0} e^{i H t}\right)$. (Note that, as it stands, $Z=1$; however, it is instructive to focus on its structure independently of its actual value.) In this expression, time evolution can be interpreted as occurring along a closed path: starting in the state described by $\rho_{0}$, the exponential $e^{-i H t}$ to the left of $\rho_{0}$ corresponds to a "forward" evolution up to the time $t$, while the exponential $e^{i H t}$ to its right corresponds to an evolution going "backward" in time. The trace $\operatorname{tr}(\cdots)$ connects, at time $t$, the forward with the backward branch of the time path and therefore it produces a closed-time-path inte- gral. Along each of these two branches, the temporal evolution can be represented in a standard way as a functional integral of an exponential weight $e^{i S}$ over suitably introduced (generally complex) integration variables, i.e., fields, $\psi_{+}(t, \mathbf{x})$ and $\psi_{-}(t, \mathbf{x})$ on the forward and backward branches, respectively. These fields are associated with the two sets of coherent states introduced as resolutions of the identity in-between two consecutive infinitesimal time evolutions in the Trotter decomposition of the unitary temporal evolution along the two branches [55, 56]. The resulting Schwinger-Keldysh action $S$ is a functional of $\psi_{ \pm}(t, \mathbf{x})$ and it is generally obtained as a temporal integral along the close path in time of a Lagrangian density. (Explicit forms of $S$ will be discussed further below, but they are not relevant for the present discussion.) By introducing different (time-dependent) sources $J_{ \pm}$for the fields $\psi_{ \pm}$on the two branches, the partition function $Z\left[J_{+}, J_{-}\right]$is no longer identically equal to 1 and its functional derivatives can be used in order to generate various time-dependent correlation functions (see, e.g., Refs. 55-57).

As we show further below in Sec. V, a system is in thermodynamic equilibrium at a temperature $T=1 / \beta$, if the corresponding Schwinger-Keldysh action is invariant under a certain transformation $\mathcal{T}_{\beta}$ which acts on the fields along the closed time path. In order to specify the form of $\mathcal{T}_{\beta}$, we focus on the dynamics of a single complex bosonic field, which is sufficiently simple but general enough to illustrate conveniently all the basic ideas. In this case, the transformation $\mathcal{T}_{\beta}$ turns out to be composed of a complex conjugation [62] of the field components $\psi_{\sigma}$ with $\sigma= \pm$, an inversion of the sign of the time variable, and a translation of the time variable into the complex plane by an amount $i \sigma \beta / 2$, i.e.,

$$
\begin{aligned}
& \mathcal{T}_{\beta} \psi_{\sigma}(t, \mathbf{x})=\psi_{\sigma}^{*}(-t+i \sigma \beta / 2, \mathbf{x}), \\
& \mathcal{T}_{\beta} \psi_{\sigma}^{*}(t, \mathbf{x})=\psi_{\sigma}(-t+i \sigma \beta / 2, \mathbf{x}) .
\end{aligned}
$$

For convenience and future reference we provide an alternative compact representation of the action of $\mathcal{T}_{\beta}$ both in the time and real space domain $(t, \mathbf{x})$ as well as in the frequency-momentum domain $(\omega, \mathbf{q})$. The convention for the Fourier transforms of the fields, conveniently collected into two spinors $\Psi_{\sigma}(t, \mathbf{x})=\left(\psi_{\sigma}(t, \mathbf{x}), \psi_{\sigma}^{*}(t, \mathbf{x})\right)^{T}$, is the following:

$$
\Psi_{\sigma}(t, \mathbf{x})=\int \frac{\mathrm{d}^{d} \mathbf{q}}{(2 \pi)^{d}} \int_{-\infty}^{+\infty} \frac{\mathrm{d} \omega}{2 \pi} e^{i(\mathbf{q} \cdot \mathbf{x}-\omega t)} \Psi_{\sigma}(\omega, \mathbf{q}) .
$$

In this relation, $d$ is the spatial dimensionality of the system, and the field spinors in the frequency-momentum domain are defined as $\Psi_{\sigma}(\omega, \mathbf{q})=\left(\psi_{\sigma}(\omega, \mathbf{q}), \psi_{\sigma}^{*}(-\omega,-\mathbf{q})\right)^{T}$. Accordingly, we can write the symmetry transformation $\mathcal{T}_{\beta}$ in the form

$$
\begin{gathered}
\mathcal{T}_{\beta} \Psi_{\sigma}(t, \mathbf{x})=\Psi_{\sigma}^{*}(-t+i \sigma \beta / 2, \mathbf{x})=\sigma_{x} \Psi_{\sigma}(-t+i \sigma \beta / 2, \mathbf{x}), \\
\mathcal{T}_{\beta} \Psi_{\sigma}(\omega, \mathbf{q})=e^{-\sigma \beta \omega / 2} \Psi_{\sigma}^{*}(\omega,-\mathbf{q})=e^{-\sigma \beta \omega / 2} \sigma_{x} \Psi_{\sigma}(-\omega, \mathbf{q}),
\end{gathered}
$$

where we introduced the Pauli matrix $\sigma_{x}=\left(\begin{array}{ll}0 & 1 \\ 1 & 0\end{array}\right)$. The transformation in real time requires evaluating the fields for complex values of the time argument, which in principle is not defined; however, the complementary representation in Fourier 
space indicates how this can be done in practice: in frequency space, the shift of time by an imaginary part $i \sigma \beta / 2$ amounts to a multiplication by a prefactor $e^{-\sigma \beta \omega / 2}$.

As usual within the Schwinger-Keldysh formalism, it is convenient to introduce what are known as classical and quantum fields. These are defined as the symmetric and antisymmetric combinations, respectively, of fields on the forward and backward branches:

$$
\phi_{c}=\frac{1}{\sqrt{2}}\left(\psi_{+}+\psi_{-}\right), \quad \phi_{q}=\frac{1}{\sqrt{2}}\left(\psi_{+}-\psi_{-}\right) .
$$

Combining these fields into spinors $\Phi_{v}(\omega, \mathbf{q})=$ $\left(\phi_{v}(\omega, \mathbf{q}), \phi_{v}^{*}(-\omega,-\mathbf{q})\right)^{T}$ - where the index $v=c, q$ distinguishes classical and quantum fields - the transformation $\mathcal{T}_{\beta}$ takes the following form, which we report here for future reference:

$$
\begin{aligned}
& \mathcal{T}_{\beta} \Phi_{c}(\omega, \mathbf{q})=\sigma_{x}\left(\cosh (\beta \omega / 2) \Phi_{c}(-\omega, \mathbf{q})-\sinh (\beta \omega / 2) \Phi_{q}(-\omega, \mathbf{q})\right) \\
& \mathcal{T}_{\beta} \Phi_{q}(\omega, \mathbf{q})=\sigma_{x}\left(-\sinh (\beta \omega / 2) \Phi_{c}(-\omega, \mathbf{q})+\cosh (\beta \omega / 2) \Phi_{q}(-\omega, \mathbf{q})\right)
\end{aligned}
$$

We anticipate and summarize here a number of properties of the equilibrium transformation $\mathcal{T}_{\beta}$, which are going to be discussed in detail in Secs. IV and V:

1. The transformation is linear, discrete and involutive, i.e., $\mathcal{T}_{\beta}^{2}=\mathbb{1}$. The last property follows straightforwardly from Eqs. (1) or (3). Concerning linearity, note in particular that the complex conjugation in Eq. (1) affects only the field variables, i.e., $\mathcal{T}_{\beta} \lambda \psi_{\sigma}(t, \mathbf{x})=$ $\lambda \psi_{\sigma}^{*}(-t+i \sigma \beta / 2, \mathbf{x})$ for $\lambda \in \mathbb{C}($ see Sec. V B).

2. $\mathcal{T}_{\beta}$ can be written as a composition $\mathcal{T}_{\beta}=\mathrm{T} \circ \mathcal{K}_{\beta}$ of a time-reversal transformation $\mathrm{T}$ and an additional transformation $\mathcal{K}_{\beta}$, which we will identify in Sec. VC as the implementation of the KMS condition within the Schwinger-Keldysh functional integral formalism.

3. $\mathcal{T}_{\beta}$ is not uniquely defined, due to a certain freedom in implementing the time-reversal transformation within the Schwinger-Keldysh functional integral formalism, as discussed in Sec. V B. However, without loss of generality, we stick to the definition provided by Eq. (1) and we comment on the alternative forms in Sec. V B.

4. The transformation $\mathcal{T}_{\beta}$ leaves the functional measure invariant, i.e., the absolute value of the Jacobian determinant associated with $\mathcal{T}_{\beta}$ is equal to one, as discussed in Sec. V D and shown in App. D.

5. The various forms of the transformation $\mathcal{T}_{\beta}$ presented above apply to the case of a system of bosons with vanishing chemical potential $\mu$. In the presence of $\mu \neq 0$, Eq. (1) becomes

$$
\begin{aligned}
& \mathcal{T}_{\beta, \mu} \psi_{\sigma}(t, \mathbf{x})=e^{\sigma \beta \mu / 2} \psi_{\sigma}^{*}(-t+i \sigma \beta / 2, \mathbf{x}), \\
& \mathcal{T}_{\beta, \mu} \psi_{\sigma}^{*}(t, \mathbf{x})=e^{-\sigma \beta \mu / 2} \psi_{\sigma}(-t+i \sigma \beta / 2, \mathbf{x}),
\end{aligned}
$$

with a consequent modification of Eq. (3), which can be easily worked out. After a transformation to the basis of classical and quantum fields according to Eq. (4), this modification amounts to shifting the frequency $\omega$ in the arguments of the hyperbolic functions in Eq. (5), i.e., to $\omega \rightarrow \omega-\mu$.
6. In taking the Fourier transforms in Eqs. (3) and (5) one implicitly assumes that the initial state of the system was prepared at time $t=-\infty$, while its evolution extends to $t=\infty$. In the following we will work under this assumption, commenting briefly on the role of an initial condition imposed at a finite time in Sec. IV C.

\section{INVARIANCE OF THE SCHWINGER-KELDYSH ACTION}

As we demonstrate further below in Sec. V, a system is in thermodynamic equilibrium if its Schwinger-Keldysh action $S$ is invariant under the transformation $\mathcal{T}_{\beta}$, i.e.,

$$
S[\Psi]=\tilde{S}\left[\mathcal{T}_{\beta} \Psi\right]
$$

where, for convenience of notation, $\Psi=\left(\psi_{+}, \psi_{+}^{*}, \psi_{-}, \psi_{-}^{*}\right)^{T}$ collects all the fields introduced in the previous section into a single vector. The tilde in $\tilde{S}$ indicates that all the parameters in $S$ which are related to external fields have to be replaced by their corresponding time-reversed values (e.g., the signs of magnetic fields have to be inverted), while in the absence of these fields the tilde may be dropped.

According to the construction of the Schwinger-Keldysh functional integral outlined at the beginning of the previous section, the action corresponding to the unitary dynamics of a closed system is completely determined by its Hamiltonian $H$. The initial state $\rho_{0}$ of the dynamics enters the functional integral as a boundary condition: if the system was prepared in the state $\rho_{0}$ at the time $t=0$, the matrix element $\left\langle\psi_{+, 0}\left|\rho_{0}\right| \psi_{-, 0}\right\rangle$, where $\left|\psi_{ \pm, 0}\right\rangle$ are coherent states, determines the (complex) weight of field configurations at the initial time with $\psi_{ \pm}(0, \mathbf{x})=\psi_{ \pm, 0}(\mathbf{x})$. In Sec. IV A, we demonstrate the invariance of the Schwinger-Keldysh action associated with a time-independent Hamiltonian dynamics under the transformation $\mathcal{T}_{\beta}$. In particular, this invariance holds for for any value of $\beta$. Interestingly enough, the SchwingerKeldysh action associated with a Hamiltonian of a simple noninteracting system — which can be diagonalized in terms of 
single-particle states - turns out to be invariant under an enhanced version of this transformation, involving possibly different values of $\beta$ for each of the single-particle states (see Sec. IV A 3). A constraint on the value of $\beta$, however, comes from the inclusion of the boundary condition for the functional integral which specifies the initial state $\rho_{0}$. Here we are interested in the stationary state of the system, which is generically reached a long time after its preparation in the state $\rho_{0}$. Hence, we assume that this was done in the distant past, i.e., at $t=-\infty$, and that the evolution of the system extends to $t=+\infty$ (cf. point 6 in Sec. III). In the construction of the Schwinger-Keldysh functional integral for a system in thermodynamic equilibrium $[55,56]$, a convenient alternative approach for specifying the appropriate boundary conditions corresponding to the initial equilibrium state $\rho_{0}$ of the system, consists in adding infinitesimal dissipative contributions to the action. Usually $[55,56]$, the form of these contributions is determined by the requirement that the Green's functions of the system are thermal with a specific temperature $T=1 / \beta$, i.e., that they obey a fluctuation-dissipation relation; once these terms are included, any reference to $\rho_{0}$ may be omitted. We demonstrate in Sec. IV B 1, that these dissipative contributions are invariant under $\mathcal{T}_{\beta}$ with exactly the same $\beta$. Hence, the thermal symmetry provides a different perspective on the construction of the Schwinger-Keldysh functional integral for a system in thermal equilibrium: while the unitary contributions are fixed by the Hamiltonian of the system, the requirement of invariance under the symmetry transformation $\mathcal{T}_{\beta}$ can be taken as the fundamental principle for specifying the structure of the dissipative terms which can occur in the action if the system is in thermodynamic equilibrium at temperature $T=1 / \beta$. We emphasize that only the simultaneous presence in the Schwinger-Keldysh action of both the Hamiltonian and the dissipative contributions yields a well-defined functional integral: the dissipative terms in the microscopic action are taken to be infinitesimally small as for an isolated system, where they merely act as a regularization which renders the functional integral finite and ensures that the bare response and correlation functions satisfy a FDR; on the other hand, if the isolated system is composed of a small subsystem of interest and a remainder which can be considered as a bath, then finite dissipative contributions emerge in the SchwingerKeldysh action of the subsystem after the bath has been integrated out. This scenario is considered in Secs. IV C and VI B. Moreover, the system can act as its own bath: in fact, one expects the effective action for the low-frequency and longwavelength dynamics of the system to contain dissipative contributions which are due to the coupling to high-frequency fluctuations. In Sec. IV B, we explicitly construct dissipative terms which comply with the thermal symmetry $\mathcal{T}_{\beta}$. In particular, we find that the noise components associated with these dissipative terms must necessarily have the form of the equilibrium Bose-Einstein distribution function, as appropriate for the bosonic fields which we are presently focussing on.

\section{A. Invariance of Hamiltonian dynamics}

The Schwinger-Keldysh action $S$ associated with the dynamics generated by a time-independent Hamiltonian $H$ can be written as the sum of a "dynamical" and a "Hamiltonian" part, $S_{\text {dyn }}$ and $S_{\mathcal{H}}$, respectively,

$$
\begin{aligned}
S & =S_{\mathrm{dyn}}+S_{\mathcal{H}}, \\
S_{\mathrm{dyn}} & =\frac{1}{2} \int_{t, \mathbf{x}}\left(\Psi_{+}^{\dagger} i \sigma_{z} \partial_{t} \Psi_{+}-\Psi_{-}^{\dagger} i \sigma_{z} \partial_{t} \Psi_{-}\right), \\
S_{\mathcal{H}} & =-\int_{t}\left(\mathcal{H}_{+}-\mathcal{H}_{-}\right),
\end{aligned}
$$

where we used the shorthand $\int_{t} \equiv \int_{-\infty}^{\infty} \mathrm{d} t, \int_{\mathbf{x}} \equiv \int \mathrm{d}^{d} \mathbf{x}$, while $\sigma_{z}=\left(\begin{array}{cc}1 & 0 \\ 0 & -1\end{array}\right)$ is the Pauli matrix. This structure of the Schwinger-Keldysh action results from the construction of the functional integral outlined at the beginning of Sec. III. In particular, the Hamiltonians $\mathcal{H}_{ \pm}$are matrix elements of the Hamiltonian operator $H$ in the basis of coherent states $\left|\psi_{ \pm}\right\rangle$, i.e., $\mathcal{H}_{\sigma}=\left\langle\psi_{\sigma}|H| \psi_{\sigma}\right\rangle /\left\langle\psi_{\sigma} \mid \psi_{\sigma}\right\rangle$, where the amplitudes $\psi_{ \pm}$of the coherent states are just the integration variables in the functional integral $[55,56]$. Henceforth we focus on the case of a bosonic many-body system with contact interaction, i.e., with Hamiltonians in Eq. (10) given by

$$
\mathcal{H}_{\sigma}=\int_{\mathbf{x}}\left(\frac{1}{2 m}\left|\nabla \psi_{\sigma}\right|^{2}+\tau\left|\psi_{\sigma}\right|^{2}+\lambda\left|\psi_{\sigma}\right|^{4}\right)
$$

Here $m$ is the mass of bosons, $\tau$ the chemical potential, and $\lambda$ parametrizes the strength of the $s$-wave two-body interaction. We consider this case because it is sufficiently general for the purpose of illustrating all basic concepts associated with the thermal symmetry and, in addition, in the classical limit it allows a direct comparison with classical stochastic models $[63,64]$, where $\phi_{c}=\left(\psi_{+}+\psi_{-}\right) / \sqrt{2}$ plays the role of a bosonic order parameter field. This point is elaborated in Sec. IV C.

Below we show that the invariance of the SchwingerKeldysh action $S$ under $\mathcal{T}_{\beta}$ is intimately related to the structure of the action, i.e., to the fact that it can be written as the sum of two terms containing, separately, only fields on the forward and backward branches.

\section{Dynamical term}

To begin with, we show that the dynamical contribution $S_{\text {dyn }}$ to the Schwinger-Keldysh action $S$ given in Eq. (9), is invariant under $\mathcal{T}_{\beta}$, i.e., that $S_{\mathrm{dyn}}\left[\mathcal{T}_{\beta} \Phi\right]=S_{\mathrm{dyn}}[\Phi]$. To this end, it is convenient to express the original fields $\left\{\psi_{ \pm}, \psi_{ \pm}^{*}\right\}$ in the so-called Keldysh basis, which is formed by the classical and quantum components $\left\{\phi_{c, q}, \phi_{c, q}^{*}\right\}$ introduced in Eq. (4). For the sake of brevity, we arrange these fields into the vector $\Phi=\left(\phi_{c}, \phi_{c}^{*}, \phi_{q}, \phi_{q}^{*}\right)^{T}$. Rewriting $S_{\text {dyn }}$ in these terms and in frequency-momentum space, we obtain $\left(\int_{\omega, \mathbf{q}} \equiv\right.$ 


$$
\begin{aligned}
& \left.\int \mathrm{d} \omega \mathrm{d}^{d} \mathbf{q} /(2 \pi)^{d+1}\right) \\
& S_{\mathrm{dyn}}\left[\mathcal{T}_{\beta} \Phi\right]=\int_{\omega, \mathbf{q}} \omega\left[\cosh ^{2}(\beta \omega / 2) \Phi_{q}^{\dagger}(\omega, \mathbf{q}) \sigma_{z} \Phi_{c}(\omega, \mathbf{q})\right. \\
& -\sinh ^{2}(\beta \omega / 2) \Phi_{c}^{\dagger}(\omega, \mathbf{q}) \sigma_{z} \Phi_{q}(\omega, \mathbf{q})+\sinh (\beta \omega / 2) \cosh (\beta \omega / 2) \\
& \left.\quad \times\left(\Phi_{c}^{\dagger}(\omega, \mathbf{q}) \sigma_{z} \Phi_{c}^{\dagger}(\omega, \mathbf{q})-\Phi_{q}^{\dagger}(\omega, \mathbf{q}) \Phi_{q}(\omega, \mathbf{q})\right)\right] . \quad(12)
\end{aligned}
$$

The combination $\Phi_{v}^{\dagger}(\omega, \mathbf{q}) \sigma_{z} \Phi_{v}(\omega, \mathbf{q})=\phi_{v}^{*}(\omega, \mathbf{q}) \phi_{v}(\omega, \mathbf{q})-$ $\phi_{v}(-\omega,-\mathbf{q}) \phi_{v}^{*}(-\omega,-\mathbf{q})$ with $v=c, q$ is an odd function of $(\omega, \mathbf{q})$, whereas $\omega \sinh (\beta \omega / 2) \cosh (\beta \omega / 2)$ is even, and therefore the integral over the product of these terms vanishes. Then, with some simple algebraic manipulation, the first two terms in Eq. (12) are recognised to be nothing but $S_{\text {dyn }}[\Phi]$, from which the invariance of $S_{\mathrm{dyn}}$ follows straightforwardly. Note that this property holds independently of the value of the parameter $\beta$ in the transformation $\mathcal{T}_{\beta}$.

\section{Hamiltonian contribution}

We consider now the transformation of the Hamiltonian contribution $S_{\mathcal{H}}$ in Eq. (10) under $\mathcal{T}_{\beta}$. First, we argue that the strictly local terms (i.e., those which do not involve spatial derivatives) in the Hamiltonian (11) are invariant under $\mathcal{T}_{\beta}$; then, we extend the argument to the case of quasilocal terms such as the kinetic energy contribution $\propto\left|\nabla \psi_{ \pm}\right|^{2}$ or non-local interactions. Consider a contribution to $S_{\mathcal{H}}$ of the form

$$
\mathcal{V}[\Psi]=\int_{t, \mathbf{x}}\left(v_{+}(t, \mathbf{x})-v_{-}(t, \mathbf{x})\right),
$$

where $v_{\sigma}(t, \mathbf{x})=\left(\psi_{\sigma}^{*}(t, \mathbf{x}) \psi_{\sigma}(t, \mathbf{x})\right)^{N}$ is a generic local contribution to the Hamiltonian $\mathcal{H}_{\sigma}$ and $N$ is an integer. In particular, for $N=1$ we obtain the term proportional to the chemical potential in Eq. (11), while for $N=2, \mathcal{V}[\Psi]$ is just the contact interaction. Since $v_{\sigma}(t, \mathbf{x})$ is real, under the transformation $\mathcal{T}_{\beta}$ [see Eq. (6)] only its time argument is shifted according to $\mathcal{T}_{\beta} v_{\sigma}(t, \mathbf{x})=v_{\sigma}(-t+i \sigma \beta / 2, \mathbf{x})$ and, taking the Fourier transform with respect to time of this relation, one eventually finds

$$
\mathcal{T}_{\beta} v_{\sigma}(\omega, \mathbf{x})=e^{-\sigma \beta \omega / 2} v_{\sigma}(-\omega, \mathbf{x}) .
$$

Accordingly, the vertex (13) is invariant under $\mathcal{T}_{\beta}$ : in fact, being local in time, its diagrammatic representation - where the fields $\psi_{\sigma}(t, \mathbf{x})$ and $\psi_{\sigma}^{*}(t, \mathbf{x})$ are represented by ingoing and outgoing lines, respectively - satisfies frequency conservation for in- and outgoing lines, as can be seen by taking the Fourier transform of each of the fields in $v_{\sigma}(t, \mathbf{x})$ individually. In particular, the frequency $\omega$ in Eq. (14) corresponds to the difference between the sums of the in- and outgoing frequencies and only the $\omega=0$ component contributes to Eq. (13). (As stated above, we assume that the time integrals in Eqs. (9), (10), and therefore (14) extend over all possible real values, i.e., we focus on the stationary state of the dynamics.) This component, however, is invariant under $\mathcal{T}_{\beta}$ as follows directly from Eq. (14), and hence the same is true for the vertex, for which $\mathcal{V}\left[\mathcal{T}_{\beta} \Psi\right]=\mathcal{V}[\Psi]$.
Clearly, the invariance of the vertex and of the dynamical term in Eq. (9) relies on the fact that vertices, which are local in time, obey frequency conservation. (Note that, as in Sec. IV A 1 , this invariance holds independently of the value of the parameter $\beta$ in $\mathcal{T}_{\beta}$.) Accordingly, one concludes that any contribution to the Hamiltonian, which is local in time and does not explicitly depend on time, is invariant. In particular, the proof of invariance presented here for the vertex in Eq. (13) can be straightforwardly extended to expressions containing spatial derivatives such as the kinetic energy $\propto\left|\nabla \psi_{ \pm}\right|^{2}$ in Eq. (11) and even to interactions which are not local in space, as long as they are local in time, as anticipated above. Note, however, that these considerations do not rule out the possible emergence upon renormalization or coarsegraining of terms which are non-local in time, as long as they are invariant under $\mathcal{T}_{\beta}$. This case is discussed further below in Sec. IV B.

\section{Enhanced symmetry for non-interacting systems}

The equilibrium transformation $\mathcal{T}_{\beta}$ presented in Sec. III involves a single parameter $\beta$. While this form is appropriate for the Gibbs ensemble describing the thermal equilibrium state of the interacting many-body system with the Hamiltonian in Eq. (11), an enhanced version of the symmetry is realized in non-interacting systems. Since these systems can be diagonalized in terms of single-particle states, they are trivially integrable. Statistically, integrable systems are described by a generalized Gibbs ensemble [6, 8, 65-71], constructed from the extensive number of conserved quantities (with possible exceptions, see, e.g., Refs. 67-70). In the case of noninteracting systems which we consider here (or, more generally, for any system that can be mapped to a non-interacting one), these integrals of motion are just the occupation numbers of single-particle states. Below we provide an example, in which the Lagrange multipliers associated with these conserved occupations enter as parameters in a generalization of the equilibrium transformation Eq. (3): more specifically, these multipliers play the role of effective inverse temperatures of the individual single-particle states. On the other hand, in non-integrable cases, the eigenstates of the Hamiltonian are not single-particle states. Then one generically expects the stationary state of the system to be in thermal equilibrium at a temperature $T=1 / \beta$, which is determined by the initial conditions of the dynamics of the system. Accordingly, the enhanced symmetry that is present in the stationary state of the non-interacting integrable system breaks down and the corresponding Schwinger-Keldysh action is invariant under a single $\mathcal{T}_{\beta}$, only for that specific value of $\beta$. This shows that the transformation $\mathcal{T}_{\beta}$ can be generalized in order to account for the appearance of a generalized Gibbs ensemble in the trivial case of a system that can be diagonalized in terms of singleparticle states. However, the question whether the generalized Gibbs ensemble emerging in the stationary states of generic integrable systems is characterized by a symmetry involving the Lagrangian multipliers associated with the respective integrals of motion as parameters, is beyond the scope of the 
present work.

As an example, let us consider bosons on a $d$-dimensional lattice with nearest-neighbour hopping and on-site interaction (i.e., the Bose-Hubbard model [72]), with Hamiltonian

$$
\begin{aligned}
H & =H_{\text {kin }}+H_{\mathrm{int}}, \\
H_{\text {kin }} & =-t \sum_{\left\langle\mathbf{1}, \mathbf{I}^{\prime}\right\rangle} a_{\mathbf{l}}^{\dagger} a_{\mathbf{l}^{\prime}}, \\
H_{\mathrm{int}} & =\frac{U}{2} \sum_{\mathbf{l}} a_{\mathbf{l}}^{\dagger} a_{\mathbf{l}}\left(a_{\mathbf{l}}^{\dagger} a_{\mathbf{l}}-1\right),
\end{aligned}
$$

where $a_{\mathbf{l}}$ is the annihilation operator for bosons on the lattice site $\mathbf{l}, t$ is the hopping matrix element between site $\mathbf{l}$ and its nearest-neighbours $\mathbf{l}^{\prime}$, while $U$ determines the strength of on-site interactions. We first consider the case $U=0$, which is trivially integrable: the kinetic energy contribution to the Hamiltonian is diagonal in momentum space and the corresponding single-particle eigenstates are the Bloch states. These are labelled by a quasi-momentum q, and in terms of creation and annihilation operators for particles in Bloch states, $a_{\mathbf{q}}^{\dagger}$ and $a_{\mathbf{q}}$ respectively, the kinetic energy can be written as

$$
H_{\mathrm{kin}}=\sum_{\mathbf{q}} \epsilon_{\mathbf{q}} a_{\mathbf{q}}^{\dagger} a_{\mathbf{q}} .
$$

Let us now consider a Schwinger-Keldysh functional integral description of the stationary state of the system. Then, the kinetic energy in Eq. (16) yields a contribution to the corresponding action which reads

$$
S_{\mathcal{H}, \text { kin }}=-\int_{t} \sum_{\mathbf{q}} \epsilon_{\mathbf{q}}\left(\psi_{\mathbf{q},+}^{*} \psi_{\mathbf{q},+}-\psi_{\mathbf{q},-}^{*} \psi_{\mathbf{q},-}\right),
$$

where $\psi_{\mathbf{q},+}$ and $\psi_{\mathbf{q},-}$ are the fields on the forward and backward branches of the closed time path respectively, expressed in the basis of Bloch states. $S_{\mathcal{H} \text {,kin }}$ is invariant under the transformation of the fields

$$
\mathcal{T}_{\beta_{\mathbf{q}}} \Psi_{\mathbf{q}, \sigma}(\omega)=e^{-\sigma \beta_{\mathbf{q}} \omega / 2} \Psi_{-\mathbf{q}, \sigma}^{*}(\omega),
$$

where, as in Eq. (3), we arrange the fields in a spinor $\Psi_{\mathbf{q}, \sigma}(\omega)=\left(\psi_{\mathbf{q}, \sigma}(\omega), \psi_{-\mathbf{q}, \sigma}^{*}(-\omega)\right)^{T}$. The crucial point is that $\beta_{\mathbf{q}}$ can be chosen to depend on the quasi-momentum $\mathbf{q}$, indicating that to each eigenstate of the system we can assign an individual "temperature" $T_{\mathbf{q}}=1 / \beta_{\mathbf{q}}$ such that the corresponding mean occupation number $n_{\mathbf{q}}=\left\langle a_{\mathbf{q}}^{\dagger} a_{\mathbf{q}}\right\rangle$ is determined by a Bose distribution with precisely this "temperature."

Let us now consider the opposite limit in which the hopping amplitude $t$ vanishes while the interaction strength $U$ is finite. The interaction energy $H_{\text {int }}$ in Eq. (15) is diagonal in the basis of Wannier states localized at specific lattice sites and the occupation numbers $\hat{n}_{\mathbf{l}}=a_{1}^{\dagger} a_{1}$ of these sites are conserved, rendering the system integrable. The generalized symmetry transformation appropriate for this case can be obtained from Eq. (18) by replacing the quasi-momentum $\mathbf{q}$ by the lattice site index $\mathbf{l}$ and by introducing a set of "local (inverse) temperatures" $T_{\mathbf{l}}\left(\beta_{\mathbf{l}}\right)$ instead of $T_{\mathbf{q}}\left(\beta_{\mathbf{q}}\right)$.
In the generic case, when both the hopping $t$ and the interaction $U$ are non-zero, the system is not integrable. Then, neither the generalized transformation Eq. (18) nor its variant with local "temperatures" are symmetries of the corresponding Schwinger-Keldysh action, showing that this case eventually admits only one single global temperature, which determines the statistical weight of individual many-body eigenstates of the system.

\section{B. Dissipative contributions in equilibrium}

The functional integral with the action $S$ in Eq. (8), as it stands, is not convergent but it can be made so by adding to $S$ an infinitesimally small imaginary (i.e., dissipative) contribution $[55,56]$. Within a renormalization-group picture, this infinitesimal dissipation may be seen as the "initial value," at a microscopic scale, of finite dissipative contributions, which are eventually obtained upon coarse graining the original action $S$ and which result in, e.g., finite lifetimes of excitations of the effective low-energy degrees of freedom. The precise form of the corresponding effective low-energy action and, in particular, of the dissipative contributions which appear therein, is strongly constrained by the requirement of invariance under $\mathcal{T}_{\beta}$ of the starting action at the microscopic scale: in fact, terms which violate this symmetry will not be generated upon coarse-graining. In the discussion below we identify those dissipative contributions to the Schwinger-Keldysh action which are invariant under $\mathcal{T}_{\beta}$. This allows us to anticipate the structure of any low-energy effective action possessing this a symmetry. Note, however, that finite dissipative terms may appear even at the microscopic scale because of, e.g., the coupling of the system to an external bath. Below we consider two instances of this case: in Sec. VIB we show that $\mathcal{T}_{\beta}$ cannot be a symmetry of the action if the system is coupled to Markovian baths and driven - a situation described by a quantum master equation. Another specific example, in which the equilibrium symmetry is realized, is the particle number non-conserving coupling of the Schwinger-Keldysh action Eq. (8) to an ohmic bath. This situation, which we discuss in Sec. IV C, is of particular interest, because its classical limit renders what is known as the dynamical model A [63] with reversible mode couplings (termed model $A^{*}$ in Ref. 64); this correspondence allows us to establish a connection with the known equilibrium symmetry of the generating functional associated with this classical stochastic dynamics.

Below we discuss dissipative terms of the action invariant under $\mathcal{T}_{\beta}$, which involve first single particles (being quadratic in the fields of the Schwinger-Keldysh action) in Sec. IV B 1, and then their interactions in Sec. IV B 2.

\section{Single-particle sector}

Dissipative contributions to the single-particle sector of the Schwinger-Keldysh action which are invariant under $\mathcal{T}_{\beta}$ take 
the form

$$
\begin{aligned}
S_{d}=i \int_{\omega, \mathbf{q}} h(\omega, \mathbf{q}) & \left(\phi_{q}^{*}(\omega, \mathbf{q}) \phi_{c}(\omega, \mathbf{q})-\phi_{q}(\omega, \mathbf{q}) \phi_{c}^{*}(\omega, \mathbf{q})\right. \\
+ & \left.2 \operatorname{coth}(\beta \omega / 2) \phi_{q}^{*}(\omega, \mathbf{q}) \phi_{q}(\omega, \mathbf{q})\right)
\end{aligned}
$$

with an arbitrary real function $h(\omega, \mathbf{q})$ which transforms under time reversal as $\tilde{h}(\omega, \mathbf{q})=h(\omega,-\mathbf{q})$. When such dissipative terms are introduced in order to regularize the SchwingerKeldysh functional integral, a typical choice for $h(\omega, \mathbf{q})$ is $h(\omega, \mathbf{q})=\epsilon[55,56]$ with $\epsilon \rightarrow 0$. This ensures that the Green's functions in the absence of interactions satisfy a fluctuationdissipation relation (we postpone the detailed discussion of such relations to Sec. VIA). The FDR for non-interacting Green's functions, together with the invariance of interactions under the transformation $\mathcal{T}_{\beta}$ shown in Sec. IV A 2, guarantees that the FDR is satisfied to all orders in perturbation theory [40].

While there are no restrictions on the form of the function $h(\omega, \mathbf{q})$, the hyperbolic cotangent $\operatorname{coth}(\beta \omega / 2)$ appearing in the last term of $S_{d}$ is uniquely fixed by the requirement of invariance under $\mathcal{T}_{\beta}$, as can be verified by following the line of argument presented in Appendix A. In particular, $S_{d}$ with a certain value of $\beta$ in the argument of $\operatorname{coth}(\beta \omega / 2)$ is invariant under $\mathcal{T}_{\beta^{\prime}}$ if and only if $\beta^{\prime}=\beta$. This shows that, remarkably, the appearance of the thermodynamic equilibrium Bose distribution function $n(\omega)=1 /\left(e^{\beta \omega}-1\right)$ at a temperature $T=1 / \beta$ in $\operatorname{coth}(\beta \omega / 2)=2 n(\omega)+1$, can be traced back to the fact that $\mathcal{T}_{\beta}$ is a symmetry of the action. Note that for simplicity we considered here only the case of vanishing chemical potential, $\mu=0$. For finite $\mu$, the frequency $\omega$ in the argument of the hyperbolic cotangent in Eq. (19) should be shifted according to $\omega \rightarrow \omega-\mu$, as we discussed in point 5 in Sec. III.

\section{Dissipative vertices}

The dissipative contributions discussed in the previous section are quadratic in the field operators and they naturally occur, e.g., when the system is coupled to a thermal bath by means of an interaction which is linear in those fields. However, this type of coupling necessarily breaks particle number conservation. The number of particles is conserved if instead the system-bath interaction term commutes with the total number of particles of the system, $N=\int_{\mathbf{x}} n(\mathbf{x})$, where $n(\mathbf{x})=\psi^{\dagger}(\mathbf{x}) \psi(\mathbf{x})$ is the local density. In other words, to ensure particle number conservation, it is necessary that the coupling terms are at least quadratic in the system operators. Accordingly, dissipative vertices appear in the Schwinger-Keldysh action after integrating out the bath degrees of freedom. Then, the requirement of invariance of these terms under $\mathcal{T}_{\beta}$ allows us to infer a priori their possible structure. In particular, we find that a frequency-independent number-conserving quartic vertex (i.e., the dissipative counterpart to the two-body interaction $\propto\left|\psi_{\sigma}\right|^{4}$ in the Hamiltonian (11)) is forbidden by the thermal symmetry.

A generic quartic vertex, which conserves the number of particles and which is local in time, can be parameterized as

$$
\begin{aligned}
& S_{d}=-i \int_{\omega_{1}, \ldots, \omega_{4}} \delta\left(\omega_{1}-\omega_{2}+\omega_{3}-\omega_{4}\right) \\
& \times \quad\left[f_{1}\left(\omega_{1}, \omega_{2}, \omega_{3}, \omega_{4}\right) \psi_{+}^{*}\left(\omega_{1}\right) \psi_{+}\left(\omega_{2}\right) \psi_{+}^{*}\left(\omega_{3}\right) \psi_{+}\left(\omega_{4}\right)\right. \\
& +f_{2}\left(\omega_{1}, \omega_{2}, \omega_{3}, \omega_{4}\right) \psi_{-}^{*}\left(\omega_{1}\right) \psi_{-}\left(\omega_{2}\right) \psi_{-}^{*}\left(\omega_{3}\right) \psi_{-}\left(\omega_{4}\right) \\
& \left.+f_{3}\left(\omega_{1}, \omega_{2}, \omega_{3}, \omega_{4}\right) \psi_{+}^{*}\left(\omega_{1}\right) \psi_{+}\left(\omega_{2}\right) \psi_{-}^{*}\left(\omega_{3}\right) \psi_{-}\left(\omega_{4}\right)\right],
\end{aligned}
$$

where $f_{1,2,3}$ are real functions; in order to simplify the notation we do not indicate the (local) spatial dependence of the fields, which is understood together with the corresponding integration in space. Conservation of particle number is ensured by the $U(1)$ invariance $\psi_{ \pm} \mapsto e^{i \alpha_{ \pm}} \psi_{ \pm}$on each contour separately, with generic phases $\alpha_{ \pm}$, while the overall $\delta$-function on the frequencies guarantees locality in time. Restrictions on the functions $f_{1,2,3}$ in the generic dissipative vertex in Eq. (20) follow from the requirements of causality [55], according to which $S_{d}$ to the action must vanish for $\psi_{+}=\psi_{-}$, and invariance of the dissipative vertex under the equilibrium transformation. These conditions are studied in detail in Appendix B. In particular, we find that they cannot be satisfied if $f_{1,2,3}$ are constant, i.e., do not depend on the frequencies. One particular choice of these functions that is compatible with the constraints is given by

$$
\begin{aligned}
f_{1}\left(\omega_{1}, \omega_{2}, \omega_{3}, \omega_{4}\right) & =f_{2}\left(\omega_{1}, \omega_{2}, \omega_{3}, \omega_{4}\right) \\
& =\left(\omega_{1}-\omega_{2}\right) \operatorname{coth}\left(\beta\left(\omega_{1}-\omega_{2}\right) / 2\right), \\
f_{3}\left(\omega_{1}, \omega_{2}, \omega_{3}, \omega_{4}\right) & =-4\left(\omega_{1}-\omega_{2}\right)\left(n\left(\omega_{1}-\omega_{2}\right)+1\right),
\end{aligned}
$$

with the Bose distribution function $n(\omega)$. It is interesting to note that, in the basis of classical and quantum fields, this corresponds to a generalization of Eq. (19) with $h(\omega, \mathbf{q})=\omega$, in which the fields are replaced by the respective densities defined as $\rho_{c}=\left(\psi_{+}^{*} \psi_{+}+\psi_{-}^{*} \psi_{-}\right) / \sqrt{2}$ and $\rho_{q}=$ $\left(\psi_{+}^{*} \psi_{+}-\psi_{-}^{*} \psi_{-}\right) / \sqrt{2}$. Another notable property of this solution is that for $\omega_{1,2} \rightarrow 0$ we have $f_{1,2}\left(\omega_{1}, \omega_{2}, \omega_{3}, \omega_{4}\right) \rightarrow 2 T$ and $f_{3}\left(\omega_{1}, \omega_{2}, \omega_{3}, \omega_{4}\right) \rightarrow-4 T$, i.e., these limits of vanishing frequencies are finite. This implies that the form of $S_{d}$ with $f_{1,2,3}$ given by Eq. (21) is to some extent universal: indeed, it should be expected to give the leading dissipative contribution to the Schwinger-Keldysh action of any number-conserving system in the low-frequency limit. At higher frequencies, other less universal solutions might also be important and one cannot make a general statement.

\section{Classical limit, detailed balance and microreversibility}

A transformation analogous to $\mathcal{T}_{\beta}$ - which becomes a symmetry in equilibrium — was previously derived for the stochastic evolution of classical statistical systems in contact with an environment, within the response functional formalism [44-47, 73-76]. This formalism allows one to determine expectation values of relevant quantities as a functional integral with a certain action known as response functional, which can also be derived from a suitable classical limit of the Schwinger-Keldysh action for quantum systems $[55,56]$. 
In these classical systems, the environment acts effectively as a source of stochastic noise over which the expectation values are taken.

Here, we show that the classical limit of $\mathcal{T}_{\beta}$ [58] yields exactly the transformation which becomes a symmetry when the classical system is at equilibrium [48]. In order to consider this limit within the Schwinger-Keldysh formalism, it is convenient to express the Schwinger-Keldysh action in Eq. (8) in terms of the classical and quantum fields $\phi_{c}$ and $\phi_{q}$, respectively, defined in Eq. (4), and to reinstate Planck's constant according to $[55,56]$

$$
S \rightarrow S / \hbar, \quad \operatorname{coth}(\beta \omega / 2) \rightarrow \operatorname{coth}(\beta \hbar \omega / 2), \quad \phi_{q} \rightarrow \hbar \phi_{q}
$$

Then, the action can be formally expanded in powers of $\hbar$ in order to take the classical limit $\hbar \rightarrow 0$, and the classical part of the Schwinger-Keldysh action is given by the contribution which remains for $\hbar=0$. Note that the limit $\hbar \rightarrow 0$ considered here is formally equivalent to approaching criticality in equilibrium at finite temperature $T=\beta^{-1}$, for which $\beta \Delta \rightarrow 0$, where $\Delta$ is the energy gap, which can be read off from the retarded Green's function (see, e.g., Ref. 77). This equivalence conforms with the expectation that quantum fluctuations generically play only a subdominant role in determining the critical behavior of quantum systems at finite temperature. In order to see the emergence of a stochastic dynamics driven by incoherent (thermal) noise from a quantum coherent dynamics, we supplement the Schwinger-Keldysh action in Eq. (8) (describing the latter) with dissipative terms arising from its coupling to a bath. For simplicity, we assume this bath to be characterized by an ohmic spectral density, while the system is assumed to have the Hamiltonian in Eq. (11). Deferring to Sec. VIB the discussion of the theoretical description of such a system-bath coupling, we anticipate here that the resulting contribution to the Schwinger-Keldysh action can be written as in Eq. (64), under the assumption that $\gamma(\omega) v(\omega)$ is linear in the frequency, i.e., $\gamma(\omega) \nu(\omega)=2 \kappa \omega$ and by choosing $L_{\sigma}(\omega) \rightarrow \psi_{\sigma}(\omega, \mathbf{q})$, with the thermal bath acting independently on each momentum mode [56]. Then, in the classical limit $\hbar \rightarrow 0$, we find

$$
\begin{aligned}
S=\int_{t, \mathbf{x}} \Phi_{q}^{\dagger}\left\{\left[\left(\sigma_{z}+i \kappa \mathbb{1}\right) i \partial_{t}\right.\right. & \left.\left.+\frac{\nabla^{2}}{2 m}\right] \Phi_{c}+i 2 \kappa T \Phi_{q}\right\} \\
& -\lambda \int_{t, \mathbf{x}}\left(\phi_{c}^{* 2} \phi_{c} \phi_{q}+\text { c.c. }\right) .
\end{aligned}
$$

This action has the form of the response functional of the equilibrium dynamical models considered in Ref. 63: it includes both a linear and a quadratic contribution in the quantum field $\phi_{q}$, but no higher-order terms. After having transformed the quadratic term into a linear one via the introduction of an auxiliary field (which is eventually interpreted as a Gaussian additive noise), this quantum field can be integrated out and one is left with an effective constraint on the dynamics of the classical field $\phi_{c}$, which takes the form of a Langevin equation; here:

$$
(i-\kappa) \partial_{t} \phi_{c}=\left(-\frac{\nabla^{2}}{2 m}+\lambda\left|\phi_{c}\right|^{2}\right) \phi_{c}+\eta
$$

where $\eta=\eta(t, \mathbf{x})$ is a (complex) Gaussian stochastic noise with zero mean $\langle\eta(t, \mathbf{x})\rangle=0$ and correlations

$$
\begin{gathered}
\left\langle\eta(t, \mathbf{x}) \eta^{*}\left(t^{\prime}, \mathbf{x}^{\prime}\right)\right\rangle=\kappa T \delta\left(t-t^{\prime}\right) \delta^{(d)}\left(\mathbf{x}-\mathbf{x}^{\prime}\right), \\
\left\langle\eta(t, \mathbf{x}) \eta\left(t^{\prime}, \mathbf{x}^{\prime}\right)\right\rangle=0 .
\end{gathered}
$$

Equation (24) describes the dynamics of the non-conserved (complex scalar) field $\phi_{c}$ without additional conserved densities, which is known in the literature as model $A$ [63]. However, as can be seen from the complex prefactor $i-\kappa$ of the time derivative on the left-hand side of Eq. (24), the dynamics is not purely relaxational as in model A, but it has additional coherent contributions, also known as reversible mode couplings [76]. The fact that the simultaneous appearance of dissipative and coherent dynamics can be described by a complex prefactor of the time derivative is specific to thermal equilibrium: in fact, dividing Eq. (24) by $i-\kappa$, the reversible and irreversible parts of the resulting Langevin dynamics are not independent of each other and in fact their coupling constants share a common ratio [46, 47, 78]. Under more general non-equilibrium conditions, however, these reversible and irreversible generators of the dynamics have different microscopic origins and no common ratio generically exists. In the present equilibrium context, however, the action Eq. (23) corresponds to model $A^{*}$ in the notion of Ref. 64, and the form of the classical transformation appropriate for this case which becomes a symmetry in equilibrium was given in Ref. 77 . This transformation emerges as the classical limit of $\mathcal{T}_{\beta}$ discussed in the previous sections [58]. In fact, for $\beta=T^{-1} \rightarrow 0$ and neglecting the contribution of the quantum fields in the transformation of the classical fields (i.e., at the leading order in $\hbar$ ), Eq. (5) becomes

$$
\begin{aligned}
& \mathcal{T}_{\beta} \Phi_{c}(t, \mathbf{x})=\sigma_{x} \Phi_{c}(-t, \mathbf{x}), \\
& \mathcal{T}_{\beta} \Phi_{q}(t, \mathbf{x})=\sigma_{x}\left(\Phi_{q}(-t, \mathbf{x})+\frac{i}{2 T} \partial_{t} \Phi_{c}(-t, \mathbf{x})\right)
\end{aligned}
$$

after a transformation back to the time and space domains. Upon identifying the classical field $\Phi_{c}$ with the physical field and $\Phi_{q}$ with the response field $\tilde{\Phi}$, according to $\Phi_{q}=i \tilde{\Phi}$, Eq. (27) takes the form of the classical symmetry introduced in Ref. 48. Note, however, that the transformation (27) is not the only form in which the equilibrium symmetry in the classical context can be expressed. In fact, the transformation of the response field $\tilde{\Phi}$ can also be expressed $[46,47]$ in terms of a functional derivative of the equilibrium distribution rather than of the time derivative of the classical field $\partial_{t} \Phi_{c}$ as in Eq. (27). The existence of these different but equivalent transformations might be related to the freedom in the definition of the response field, which is introduced in the theory as an auxiliary variable in order to enforce the dynamical constraint represented by the Langevin equation [46, 47, 76, 79] such as Eq. (24). This implies [79] that the related action acquires the so-called Slavnov-Taylor symmetry. As far as we know, the consequences of this symmetry have not been thoroughly investigated in the classical case and its role for quantum dynamics surely represents an intriguing issue for future studies.

We emphasize the fact that the derivation of the symmetry in the classical case involves explicitly the equilibrium prob- 
ability density [46, 47]. Indeed, the response functional contains an additional contribution from the probability distribution of the value of the fields at the initial time, after which the dynamics is considered. This term generically breaks the time-translational invariance of the theory [46, 47], unless the initial probability distribution is the equilibrium one. Accordingly, when the classical equilibrium symmetry $\mathcal{T}_{\beta}$ is derived under the assumption of time-translational invariance, its expression involves also the equilibrium distribution. In the quantum case discussed in the previous sections, instead, time-translational symmetry was implicitly imposed by extending the time integration in the action from $-\infty$ to $+\infty$, which is equivalent to the explicit inclusion of the initial condition (in the form of an initial density matrix) and makes the analysis simpler, though with a less transparent interpretation from the physical standpoint.

Although in classical systems this equilibrium symmetry takes (at least) two different but equivalent forms due to the arbitrariness in the definition of the response functional mentioned above, it can always be traced back to the condition of detailed balance [46-48]. Within this context, detailed balance is defined by the requirement that the probability of observing a certain (stochastic) realization of the dynamics of the system equals the probability of observing the time-reversed realization, and therefore it encodes the notion of microreversibility. This condition guarantees the existence and validity of fluctuation-dissipation relations, which can be proved on the basis of this symmetry. In addition, detailed balance constrains the form that the response functional can take as well as the one of the equilibrium probability distribution for this stochastic process.

The situation in the quantum case appears to be significantly less clear. In fact, a precise and shared notion of quantum detailed balance and quantum microreversibility is seemingly lacking. The first attempt to introduce a principle of quantum detailed balance dates back to Ref. 80, where it was derived from a condition of microreversibility in the context of Markovian quantum dynamics described by a Lindblad master equation. The mathematical properties of these conditions were subsequently studied in detail (see, e.g., Refs. 81-85) and were shown to constrain the form of the Lindblad superoperator in order for it to admit a Gibbs-like stationary density matrix. However, even when this occurs, these operators are not able to reproduce the KMS condition and the fluctuationdissipation relations because of the underlying Markovian approximation, as we discuss in Sec. VIB.

The notion of microreversibility in quantum systems appears to have received even less attention, as well as its connection with some sort of reversibility expressed in terms of the probability of observing certain "trajectories" and their time-reversed ones. The definition proposed in Ref. 80 (also discussed in Ref. 86) appears to be a natural generalization of the notion in the classical case, as it relates the correlation of two operators evaluated at two different times with the correlation of the time-reversed ones. However, to our knowledge, the relationship between this condition and thermodynamic equilibrium has never been fully elucidated. Although addressing these issues goes well beyond the scope of the present paper, they surely represent an interesting subject for future investigations.

\section{EQUIVALENCE BETWEEN THE SYMMETRY AND THE KMS CONDITION}

In this section we show that the invariance of the Schwinger-Keldysh action of a certain system under $\mathcal{T}_{\beta}$ (as specified in Sec. IV) is equivalent to having multi-time correlation functions of the relevant fields which satisfy the KMS condition $[42,43]$. As the latter can be considered as the defining property of thermodynamic equilibrium, this shows that the same applies to the invariance under the equilibrium symmetry.

The KMS condition involves both the Hamiltonian generator of dynamics and the thermal nature of the density matrix which describes the stationary state of the system: heuristically this condition amounts to requiring that the manybody Hamiltonian which determines the (canonical) population of the various energy levels is the same as the one which rules the dynamics of the system. The equivalence proved here allows us to think of the problem from a different perspective: taking the invariance under $\mathcal{T}_{\beta}$ as the fundamental property and observing that any time-independent Hamiltonian respects it, we may require it to hold at any scale, beyond the microscopic one governed by reversible Hamiltonian dynamics alone. In particular, upon coarse graining within a renormalization-group framework, only irreversible dissipative terms which comply with the symmetry (such as those discussed in Sec. IV B) can be generated in stationary state and the hierarchy of correlation functions respect thermal fluctuation-dissipation relations. The validity of KMS conditions (and therefore of the symmetry $\mathcal{T}_{\beta}$ ) hinges on the whole system being prepared in a canonical density matrix $\rho$. Accordingly, if the system is described by a microcanonical ensemble, the KMS condition holds only in a subsystem of it, which is expected to be described by a canonical reduced density matrix. Equivalently, this means that, in a microcanonical ensemble, only suitable local observables satisfy this condition. In the case of quantum many-body systems evolving from a pure state, an additional restriction on the class of observables emerges due to the fact that, if thermalization occurs as conjectured by the eigenstate thermalization hypothesis (ETH) [8, 87, 88], the microcanonical ensemble is appropriate only if the observable involves the creation and annihilation of a small number of particles (low order correlation functions). This was shown to be also the case for FDRs [89, 90]: however, as pointed out above, the thermal symmetry implies the validity of FDRs involving an arbitrary number of particles, which leads to the conclusion that it does not apply to an isolated system thermalizing via the ETH. In other words, the thermal symmetry implies that the whole density matrix takes the form of a Gibbs ensemble, while in thermalization according to the ETH, only finite subsystems are thermalized by the coupling to the remainder of the system, which acts as a bath. Thus we see how Hamiltonian dynamics favors thermal stationary states (with density matrix 
$\rho$ proportional to $e^{-\beta H}$ ) over arbitrary functionals $\rho=\rho(H)$. One explicit technical advantage of this perspective based on symmetry is that it allows us to utilize the toolbox of quantum field theory straightforwardly and to study the implications of $\mathcal{T}_{\beta}$ being a symmetry; this is exemplified here by considering the associated Ward-Takahashi identities and by showing the absence of this symmetry in dynamics described by Markovian quantum master equations in Sec. VIB. We also note that the presence of this symmetry provides a criterion for assessing the equilibrium nature of a certain dynamics by inspecting only the dynamic action functional, instead of the whole hierarchy of fluctuation-dissipation relations. In addition, this symmetry may be present in the actions of open systems with both reversible and dissipative terms.

In the following, we consider a quantum system with unitary dynamics generated by the (time-independent) Hamiltonian $H$, which is in thermal equilibrium at temperature $T=\beta^{-1}$ and therefore has a density matrix $\rho=e^{-\beta H} / \operatorname{tr} e^{-\beta H}$. The KMS condition relies on the observation that for an operator in the Heisenberg representation $A(t)=e^{i H t} A e^{-i H t}$, one has

$$
A(t) \rho=\rho A(t-i \beta)
$$

(for simplicity we do not include here a chemical potential, but at the end of the discussion we indicate how to account for it). This identity effectively corresponds, up to a translation of the time by an imaginary amount, to exchanging the order of the density matrix and of the operator $A$ and therefore, when Eq. (28) is applied to a multi-time correlation function, it inverts the time order of the involved times, which can be subsequently restored by means of the quantum-mechanical timereversal operation. Hence, the quantum-mechanical time reversal naturally appears as an element of the equilibrium symmetry $\mathcal{T}_{\beta}$, while external fields have to be transformed accordingly, as indicated in Eq. (7). The application of time reversal yields a representation of the KMS condition which can be readily translated into the Schwinger-Keldysh formalism, as was noted in Refs. 38-40. In particular, it results in an infinite hierarchy of generalized multi-time quantum fluctuationsdissipation relations which include the usual FDR for twotime correlation and response functions of the bosonic fields as a special case (see Ref. 40 and Sec. VI A). One of the main points of this work is that these FDRs can also be regarded as the Ward-Takahashi identities associated with the invariance of the Schwinger-Keldysh action $S$ under the discrete symmetry [91] $\mathcal{T}_{\beta}$ and that, conversely, the full hierarchy of FDRs implies the invariance of $S$ under $\mathcal{T}_{\beta}$.

The argument outlined below, which shows the equivalence between the KMS condition and the thermal symmetry, involves several steps: as a preliminary we review in Secs. V A and V B how time-ordered and anti-time-ordered correlation functions can be expressed using the Schwinger-Keldysh technique and we specify how these correlation functions transform under quantum mechanical time reversal. We apply these results to the KMS condition in Sec. V C: first we discuss its generalization to multi-time correlation functions and then we translate such a generalization into the SchwingerKeldysh formalism. This part proceeds mainly along the lines of Ref. 40, with some technical differences. Finally, we establish the equivalence between the resulting hierarchy of FDRs and the thermal symmetry at the end of Sec. V C.

\section{A. Multi-time correlation functions in the Schwinger-Keldysh formalism}

a. Two-time correlation functions. Let us first consider a two-time correlation function

$$
\left\langle A\left(t_{A}\right) B\left(t_{B}\right)\right\rangle \equiv \operatorname{tr}\left(A\left(t_{A}\right) B\left(t_{B}\right) \rho\right)
$$

between two generic operators $A$ and $B$ (in the following we are particularly interested in considering the case in which $A$ and $B$ are the field operators $\psi(\mathbf{x})$ or $\psi^{\dagger}(\mathbf{x})$ at positions $\mathbf{x}=\mathbf{x}_{A}$ and $\mathbf{x}=\mathbf{x}_{B}$ ) evaluated at different times $t_{A}$ and $t_{B}$, respectively, in a quantum state described by the density matrix $\rho$. We assume that the dynamics of the system is unitary and generated by the Hamiltonian $H$. Then, the Heisenberg operator $A$ at time $t_{A}$ is related to the Schrödinger operator at a certain initial time $t_{i}<t_{A}$ via

$$
A\left(t_{A}\right)=e^{i H\left(t_{A}-t_{i}\right)} A e^{-i H\left(t_{A}-t_{i}\right)},
$$

with an analogous relation for $B$.

The two-time correlation function can be represented within the Schwinger-Keldysh formalism as (see Appendix C)

$$
\begin{aligned}
\left\langle A\left(t_{A}\right) B\left(t_{B}\right)\right\rangle & =\left\langle A_{-}\left(t_{A}\right) B_{+}\left(t_{B}\right)\right\rangle \\
& \equiv \int \mathcal{D}[\Psi] A_{-}\left(t_{A}\right) B_{+}\left(t_{B}\right) e^{i S[\Psi]},
\end{aligned}
$$

irrespective of the relative order of the times $t_{A}$ and $t_{B}$. Here, the functional integral is taken over the fields $\Psi=$ $\left(\psi_{+}, \psi_{+}^{*}, \psi_{-}, \psi_{-}^{*}\right)^{T}$, and the exponential weight with which a specific field configuration contributes to the integral is determined by the Schwinger-Keldysh action $S[\Psi]$. In the following, by $O_{+/-}$we indicate that a certain operator $O$ has been evaluated in terms of the fields $\psi_{ \pm}$defined on the forward/backward branch of the temporal contour associated with the Schwinger-Keldysh formalism (see, e.g., Refs. 55 and 56).

b. Multi-time correlation functions. We define multitime correlation functions in terms of time-ordered and antitime-ordered products of operators

$$
\begin{aligned}
& A\left(t_{A, 1}, \ldots, t_{A, N}\right)=a_{1}\left(t_{A, 1}\right) a_{2}\left(t_{A, 2}\right) \cdots a_{N}\left(t_{A, N}\right), \\
& B\left(t_{B, 1}, \ldots, t_{B, M}\right)=b_{M}\left(t_{B, M}\right) b_{M-1}\left(t_{B, M-1}\right) \cdots b_{1}\left(t_{B, 1}\right),
\end{aligned}
$$

for $t_{i}<t_{A, 1}<\cdots<t_{A, N}<t_{f}$ and $t_{i}<t_{B, 1}<\cdots<$ $t_{B, M}<t_{f}$, where $t_{f}$ is an arbitrarily chosen largest time. Here, $\left\{a_{n}, b_{m}\right\}_{n, m}$ are bosonic field operators. The specific sequence of time arguments in $A$ and $B$ (increasing and decreasing from left to right, respectively) leads to a time-ordering on the Schwinger-Keldysh contour: indeed, as we show in Appendix $\mathrm{C}$, the multi-time correlation function can be expressed as a Schwinger-Keldysh functional integral in the form

$$
\begin{aligned}
& \left\langle A\left(t_{A, 1}, \ldots, t_{A, N}\right) B\left(t_{B, 1}, \ldots, t_{B, M}\right)\right\rangle \\
& =\left\langle B_{+}\left(t_{B, 1}, \ldots, t_{B, M}\right) A_{-}\left(t_{A, 1}, \ldots, t_{A, N}\right)\right\rangle .
\end{aligned}
$$


c. Anti-time-ordered correlation functions. Not only time-ordered correlation functions such as Eq. (33) can be expressed in terms of functional integrals, but also correlation functions which are anti-time-ordered and which, e.g., are obtained by exchanging the positions of $A\left(t_{A, 1}, \ldots, t_{A, N}\right)$ and $B\left(t_{B, 1}, \ldots, t_{B, M}\right)$ on the 1.h.s. of Eq. (33). The construction of the corresponding functional integral can be accomplished with a few straightforward modifications to the procedure summarized in Appendix C (and presented, e.g., in Refs. 55 and 56). In a stationary state one has $[\rho, H]=0$ and all the Heisenberg operators on the 1.h.s. of Eq. (33) can be related to the Schrödinger operators at a later time $t_{f}$. Then one finds

$$
\begin{aligned}
\left\langleB \left( t_{B, 1}, \ldots,\right.\right. & \left.\left.t_{B, M}\right) A\left(t_{A, 1}, \ldots, t_{A, N}\right)\right\rangle \\
& =\left\langle A_{+}\left(t_{A, 1}, \ldots, t_{A, N}\right) B_{-}\left(t_{B, 1}, \ldots, t_{B, M}\right)\right\rangle_{S_{b}},
\end{aligned}
$$

where the action $S_{b}$ describes the backward evolution and it is related to the action $S$ which enters the forward evolution in Eq. (33) simply by a global change of $\operatorname{sign} S_{b}=-S$.

\section{B. Quantum-mechanical time reversal}

In this section we first recall some properties of the quantum-mechanical time reversal operation $T$ [41] and then discuss its implementation within the Schwinger-Keldysh formalism. $T$ is an antiunitary operator, i.e., it is antilinear (such that $\mathrm{T} \lambda|\psi\rangle=\lambda^{*} \mathrm{~T}|\psi\rangle$ for $\left.\lambda \in \mathbb{C}\right)$ and unitary $\left(\mathrm{T}^{\dagger}=\mathrm{T}^{-1}\right)$. Scalar products transform under antiunitary transformations into their complex conjugates, i.e., $\langle\psi|A| \phi\rangle=\langle\tilde{\psi}|\tilde{A}| \tilde{\phi}\rangle^{*}$, where we denote by $|\tilde{\psi}\rangle=\mathrm{T}|\psi\rangle$ and $\tilde{A}=\mathrm{T} A \mathrm{~T}^{\dagger}$ the state and the Schrödinger operator obtained from the state $|\psi\rangle$ and the operator $A$, respectively, after time reversal. Accordingly, expressing the trace of an operator in a certain basis $\left\{\left|\psi_{n}\right\rangle\right\}_{n}$, one finds

$$
\operatorname{tr} A=\sum_{n}\left\langle\psi_{n}|A| \psi_{n}\right\rangle=\sum_{n}\left\langle\tilde{\psi}_{n}|\tilde{A}| \tilde{\psi}_{n}\right\rangle^{*}=(\operatorname{tr} \tilde{A})^{*} .
$$

In the last equality we used the fact that, due to the unitarity of T, also the time-reversed set $\left\{\left|\tilde{\psi}_{n}\right\rangle\right\}_{n}$ forms a basis. For future convenience, we shall define the Heisenberg representation of time-reversed operators such that it coincides with the Schrödinger one at time $-t_{f}$, i.e., we set

$$
\tilde{A}\left(t_{A}\right)=e^{i \tilde{H}\left(t_{A}+t_{f}\right)} \tilde{A} e^{-i \tilde{H}\left(t_{A}+t_{f}\right)}
$$

Note that this is distinct from the Heisenberg representation defined in Eq. (30), which coincides with the Schrödinger one only at time $t_{i}$. In order to simplify the notation, we shall not distinguish these two different Heisenberg representations, assuming implicitly that the latter and the former are used, respectively, for operators and their time-reversed ones, such that $A\left(t_{i}\right)=A$ while $\tilde{A}\left(-t_{f}\right)=\tilde{A}$.

Let us now study the effect of time reversal on the generic multi-time correlation function in Eq. (33). Due to translational invariance in time, the time arguments of the operators
$A$ and $B$ can be shifted by $t_{i}-t_{f}$ without affecting the correlation function. Then, by using Eqs. (35) and (36), one has

$$
\begin{aligned}
\left\langle A\left(t_{A, 1}, \ldots, t_{A, N}\right) B\left(t_{B, 1}, \ldots, t_{B, M}\right)\right\rangle \\
\quad=\left\langle\tilde{A}\left(-t_{A, 1}, \ldots,-t_{A, N}\right) \tilde{B}\left(-t_{B, 1}, \ldots,-t_{B, M}\right)\right\rangle_{\tilde{\rho}}^{*} \\
\quad=\left\langle\tilde{B}^{\dagger}\left(-t_{B, 1}, \ldots,-t_{B, M}\right) \tilde{A}^{\dagger}\left(-t_{A, 1}, \ldots,-t_{A, N}\right)\right\rangle_{\tilde{\rho}},
\end{aligned}
$$

where the subscript in $\langle\cdots\rangle_{\tilde{\rho}}$ indicates that the expectation value is taken with respect to the time-reversed density operator $\tilde{\rho} \equiv \mathrm{T} \rho \mathrm{T}^{\dagger}$, which is time-independent. The expectation value on the r.h.s. of Eq. (37) is anti-time ordered and therefore it can be rewritten as a Schwinger-Keldysh functional integral by using Eq. (34). The 1.h.s., instead, is time-ordered and therefore it can be expressed as in Eq. (33), such that Eq. (37) becomes

$$
\begin{aligned}
& \left\langle B_{+}\left(t_{B, 1}, \ldots, t_{B, M}\right) A_{-}\left(t_{A, 1}, \ldots, t_{A, N}\right)\right\rangle \\
& \quad=\left\langle\tilde{A}_{+}^{*}\left(-t_{A, 1}, \ldots,-t_{A, N}\right) \tilde{B}_{-}^{*}\left(-t_{B, 1}, \ldots,-t_{B, M}\right)\right\rangle_{\tilde{S}_{b}},
\end{aligned}
$$

where the subscript $b$ in $\tilde{S}_{b}$ indicates that the sign of the action which describes the Hamiltonian evolution on the r.h.s. of this relation has been reversed, as explained below Eq. (34). The time-reversed action $\tilde{S}$ differs from the action $S$ associated with $H$ which enters (implicitly, cf. Eq. (31)) Eq. (33) because in $\tilde{S}$ the time evolution is generated by $\tilde{H}$, the initial state is the time-reversed density matrix $\tilde{\rho}$, and the integration over time extends from $-t_{f}$ to $-t_{i}$. This latter difference becomes inconsequential as $t_{i} \rightarrow-\infty$ and $t_{f} \rightarrow \infty$.

Let us now consider the case in which $A$ and $B$ are products of the bosonic field operators $\psi$ and $\psi^{\dagger}$, such that $A_{ \pm}$and $B_{ \pm}$ involve the corresponding products of $\psi_{ \pm}$and their complex conjugates. As there are no further restrictions on $A$ and $B$, the 1.h.s. of Eq. (38) can be generically indicated as $\langle O[\Psi]\rangle$, where $O[\Psi]$ is the product of various fields on the SchwingerKeldysh contour corresponding to $B_{+}(\ldots) A_{-}(\ldots)$ which, according to the notation introduced in Sec. III, are collectively indicated by $\Psi=\left(\psi_{+}, \psi_{+}^{*}, \psi_{-}, \psi_{-}^{*}\right)^{T}$. With this shorthand notation, Eq. (38) can be cast in the form

$$
\langle O[\Psi]\rangle=\langle O[T \Psi]\rangle_{\tilde{S}_{b}},
$$

where the transformation

$$
\mathrm{T} \Psi_{\sigma}(t, \mathbf{x})=\Psi_{-\sigma}^{*}(-t, \mathbf{x}),
$$

implements the quantum-mechanical time reversal within the Schwinger-Keldysh formalism. (With a slight abuse of notation, the same symbol $\mathrm{T}$ is used to indicate here both the quantum-mechanical time-reversal operator introduced above and the transformation of fields on the Schwinger-Keldysh contour in Eqs. (39) and (40).) In Eq. (40) we took into account that the bosonic field operators in the Schrödinger picture and in the real-space representation are time-reversal invariant, i.e., $\tilde{\psi}(\mathbf{x})=\mathrm{T} \psi(\mathbf{x}) \mathrm{T}^{\dagger}=\psi(\mathbf{x})$, which allows us to drop the tilde on the transformed field on the r.h.s. of Eq. (40). However, we note that in the last line of Eq. (37) the Hermitean adjoint operators of those on the 1.h.s. appear and this is the reason why both the r.h.s. of Eq. (38) and the transformation prescription Eq. (40) involve complex conjugation 
of the fields. Note that the time-reversal transformation in Eq. (40) is actually linear, i.e., the complex conjugation affects only the fields variables and not possible complex prefactors. This follows again from the last line of Eq. (37): the combination of two antilinear transformations (time reversal and Hermitean conjugation) results into a linear one.

Let us mention that while Eq. (38) follows from the second line of Eq. (37), one could have equivalently taken its first line as the starting point for deriving a Schwinger-Keldysh timereversal transformation. Then one would have been lead to a different implementation $\mathrm{T}^{\prime}$ of time reversal:

$$
\mathrm{T}^{\prime} \Psi_{\sigma}(t, \mathbf{x})=\Psi_{\sigma}(-t, \mathbf{x})
$$

with an additional overall complex conjugation of the correlation function. In some sense, the transformation $\mathrm{T}^{\prime}$ is closer to the common way of representing the quantum-mechanical time reversal than $\mathrm{T}$ is, as it amounts to a mapping $t \mapsto-t$ and $i \mapsto-i$ [41]. For our purposes, however, $\mathrm{T}$ is of main interest, since it is part of the equilibrium transformation as we describe below.

We finally note that an alternative implementation of the time-reversal transformation is based on the observation that the forward and backward branches of the closed-time path integral are actually equivalent if the dynamics of a system is time-reversal invariant (TRI) [55]. More specifically, this means that the Schwinger-Keldysh action is invariant - up to a global change of sign - upon exchanging the corresponding fields, i.e., $S\left[\psi_{+}, \psi_{-}\right]=-S\left[\psi_{-}, \psi_{+}\right]$. This transformation is partly recovered in Eqs. (39) and (40): in fact, T in Eq. (40) involves an exchange of the contour indices, while the corresponding global change of sign in the action is indicated on the r.h.s. of Eq. (39) by the subscript $\tilde{S}_{b}$ (cf. the definition of $S_{b}$ below Eq. (34)). However, the time reversal transformation $\mathrm{T}$ in Eq. (40) - derived from the quantum-mechanical time-reversal operation - additionally involves both complex conjugation and the time inversion $t \mapsto-t$.

\section{KMS condition and generalized fluctuation-dissipation relations}

The discussion of the previous sections about multi-time correlation functions and the time-reversal transformation provides the basis for the formulation of the KMS condition for multi-time correlation functions and of its representation in terms of Schwinger-Keldysh functional integrals. For the specific case of a four-time correlation function, the KMS condition is pictorially illustrated in Fig. 1. As described in the caption, panel (a) summarizes the convention as far as the forward/backward contours are concerned. Panel $(b)$, instead, refers to the KMS condition which, as anticipated after Eq. (28), involves a contour exchange of the multi-time operators $A$ and $B$. This exchange is the reason why the arrows in the second equality in Fig. $1(b)$ are reversed, since both $A$ and $B$ turn out to be anti-time ordered when moved to the opposite contour. The appropriate time-ordering can be restored by means of the quantum-mechanical time reversal transformation introduced in Sec. V B, as indicated by the third equality in the figure. This is a crucial step, because only time-ordered correlation functions can be directly translated into the functional integral by means of the usual Trotter decomposition, which makes the time-reversal transformation indispensable in the construction. However, this does not mean that properties related to equilibrium conditions such as fluctuation-dissipation relations are fulfilled only if the Hamiltonian is invariant under the time-reversal transformation. Indeed, it turns out that multi-time FDRs always involve both the Hamiltonian and its time-reversed counterpart [40], while as we show in Sec. VIA the FDR for two-time functions can be stated without reference to the time-reversed Hamiltonian, even if the Hamiltonian is not TRI.

The KMS condition for a two-time function reads [92]

$$
\left\langle A\left(t_{A}\right) B\left(t_{B}\right)\right\rangle=\left\langle B\left(t_{B}-i \beta / 2\right) A\left(t_{A}+i \beta / 2\right)\right\rangle .
$$

This relation can be proven by writing down explicitly the expectation value on the 1.h.s. with $\rho=e^{-\beta H} / \operatorname{tr} e^{-\beta H}$ and by inserting the definition of the Heisenberg operators reported in Eq. (30). The generalization of this procedure to the case of multi-time correlation functions is straightforward and yields

$$
\begin{aligned}
& \left\langle A\left(t_{A, 1}, \ldots, t_{A, N}\right) B\left(t_{B, 1}, \ldots, t_{B, M}\right)\right\rangle \\
& =\left\langle B\left(t_{B, 1}-i \beta / 2, \ldots, t_{B, M}-i \beta / 2\right)\right. \\
& \left.\quad \times A\left(t_{A, 1}+i \beta / 2, \ldots, t_{A, N}+i \beta / 2\right)\right\rangle .
\end{aligned}
$$

The real parts of the time variables on the r.h.s. of this equation are such that the corresponding product of operators is anti-time-ordered (see Fig. 1). According to their definition in Eq. (32), $A$ and $B$ correspond to products of operators with, respectively, decreasing and increasing time arguments from right to left. Consequently, Eq. (43) can be expressed as a functional integral by using Eqs. (33) and (34) on the 1.h.s. and r.h.s., respectively. The presence of an imaginary part in the time arguments of Eq. (43) does not constitute a problem: in fact, the functional integral along the vertical parts of the time path in Fig. 1 can be constructed by the same method as the horizontal parts, which is summarized in Sec. V A. Hence, we find

$$
\begin{aligned}
\left\langle B_{+}\left(t_{B, 1}, \ldots, t_{B, M}\right) A_{-}\left(t_{A, 1}, \ldots, t_{A, N}\right)\right\rangle & \\
= & \left\langle A_{+}\left(t_{A, 1}+i \beta / 2, \ldots, t_{A, N}+i \beta / 2\right)\right. \\
& \left.\quad \times B_{-}\left(t_{B, 1}-i \beta / 2, \ldots, t_{B, M}-i \beta / 2\right)\right\rangle_{S_{b}} .
\end{aligned}
$$

As we did in Eq. (39) for the case of quantum-mechanical time-reversal, we may rewrite this equation in the form

$$
\langle O[\Psi]\rangle=\left\langle O\left[\mathcal{K}_{\beta} \Psi\right]\right\rangle_{S_{b}},
$$

where we define

$$
\mathcal{K}_{\beta} \Psi_{\sigma}(t)=\Psi_{-\sigma}(t-i \sigma \beta / 2) .
$$

This transformation $\mathcal{K}_{\beta}$ can be combined with the quantum mechanical time reversal T defined in Eq. (40) in order to express the equilibrium transformation $\mathcal{T}_{\beta}$ as $\mathcal{T}_{\beta}=\mathrm{T} \circ \mathcal{K}_{\beta}$. [93] By using Eq. (39) on the r.h.s. of Eq. (45), one concludes that the KMS condition implies

$$
\langle O[\Psi]\rangle=\left\langle O\left[\mathcal{T}_{\beta} \Psi\right]\right\rangle_{\tilde{S}},
$$


(a)

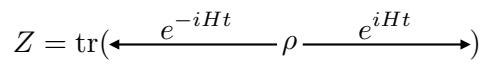

(b)

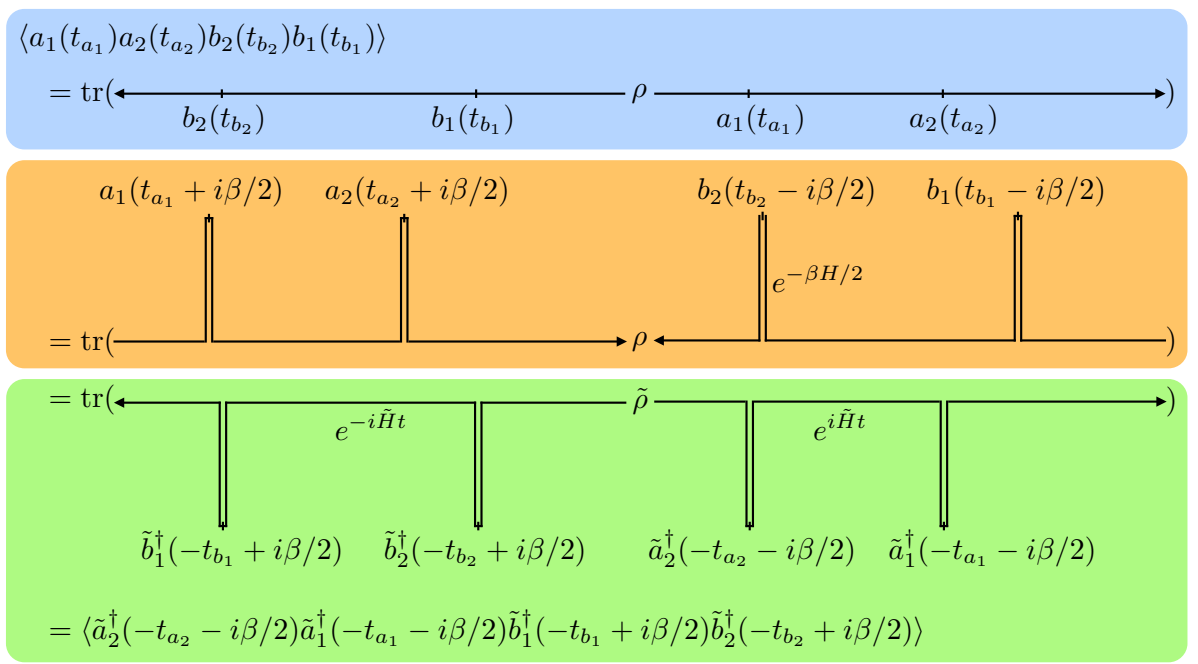

Figure 1. (Color online) (a) Schematic representation of the Schwinger-Keldysh partition function [55, 56]. The time evolution of the density matrix $\rho(t)=e^{-i H t} \rho e^{i H t}$ can be represented by introducing two time lines to the left and right of $\rho$. These time lines correspond to the + and parts of the Schwinger-Keldysh contour, respectively. (b) Schematic representation of the KMS condition for a four-time correlation function $\left\langle a_{1}\left(t_{a, 1}\right) a_{2}\left(t_{a, 2}\right) b_{2}\left(t_{b, 2}\right) b_{1}\left(t_{b, 1}\right)\right\rangle$ with $t_{a, 1}<t_{a, 2}$ and $t_{b, 1}<t_{b, 2}$, where $a_{1,2}$ and $b_{1,2}$ are bosonic field operators. As illustrated by the first equality (light blue box), this correlation function is properly time-ordered and therefore it can be directly represented within the Schwinger-Keldysh formalism with the operators $a_{1,2}$ and $b_{1,2}$ evaluated along the - and + contours, respectively. The thermal density matrix $\rho=e^{-\beta H} / \operatorname{tr} e^{-\beta H}$ can be first split into the products of $e^{-\beta H / 2} \times e^{-\beta H / 2}$ and then these two factors can be moved in opposite directions along the two time lines, with the effect of adding $+i \beta / 2$ and $-i \beta / 2$ to the time arguments of $a_{1,2}$ and $b_{1,2}$, respectively. After these two factors have been moved to the end of the timelines, due to the cyclic property of the trace, they combine as represented by the second equality (orange box), where the time lines now take detours into the complex plane and the overall time order is effectively reversed as indicated by the arrows, which converge towards $\rho$ instead of departing from it as in the case of sketch $(a)$ or of the first equality of sketch $(b)$. The original time ordering can be then restored by means of the time-reversal operation T, upon application of which operators are replaced by time-reversal transformed ones, $\tilde{\rho}=\mathrm{T} \rho \mathrm{T}^{\dagger}$ etc., and the signs of time variables are reversed. In addition, due to the anti-unitarity of T one has to take the Hermitian adjoint of the expression inside the trace. As a result, the order of operators is inverted and one obtains the third equality (green box) which is again properly time ordered. This construction can be generalized to arbitrary correlation functions, leading to Eq. (47).

which indeed provides a generalized FDR for the correlation function $\langle O[\Psi]\rangle[40]$. For various choices of the observable $O[\Psi]$ we obtain the full hierarchy of multi-time FDRs, which contains as a special case the usual FDR for two-time functions (see Sec. VI A). Before demonstrating that this hierarchy is actually equivalent to the invariance of the SchwingerKeldysh action as expressed by Eq. (7), several remarks are in order:

1. Although by means of the time-reversal transformation $\mathrm{T}$ we were able to restore the time ordering in Eq. (44), Eq. (47) still involves the time-reversed action $\tilde{S}$ and not the original action $S$. However, in practice it will typically be clear how $\tilde{S}$ can be obtained from $S$, e.g., by reversing the signs of external magnetic fields. In the absence of fields which break time-reversal invariance the tilde in Eq. (47) may be dropped, i.e., $\tilde{S}=S$.

2. Equation (47) provides a generalized FDR expressed in terms of products $O[\Psi]$ of fields on the forward and backward branches, which we collected in the fourcomponent vector $\Psi=\left(\psi_{+}, \psi_{+}^{*}, \psi_{-}, \psi_{-}^{*}\right)^{T}$. A more fa- miliar formulation of FDRs is provided in terms of classical and quantum fields $\left(\Phi_{c}\right.$ and $\Phi_{q}$, see Eq. (4)), which allow one to identify correlations functions (i.e., expectation values involving only classical fields) and response functions or susceptibilities (expectation values involving both classical and quantum fields). FDRs provide relations between correlation and response functions. In order to express the KMS condition in Eq. (47) in terms of the classical and quantum fields $\Phi_{c}$ and $\Phi_{q}$ (or, alternatively, of $\Phi=\left(\phi_{c}, \phi_{c}^{*}, \phi_{q}, \phi_{q}^{*}\right)^{T}$ ), we note that they are linearly related to $\Psi_{+}$and $\Psi_{-}$and therefore a generic product $O[\Phi]$ of such fields can be expressed as a linear combination (with real coefficients) of products $O_{i}[\Psi]$. According to Eq. (47), the expectation value of such a combination and therefore $\langle O[\Phi]\rangle$ can be expressed as on its r.h.s. in terms of the same linear combination of $\left\langle O_{i}\left[\mathcal{T}_{\beta} \Psi\right]\right\rangle_{\tilde{S}}$; since the transformation $\mathcal{T}_{\beta}$ is linear, it immediately follows that this linear combination is nothing but $\left\langle O\left[\mathcal{T}_{\beta} \Phi\right]\right\rangle_{\tilde{S}}$, where the explicit form of the transformation of the components of the field $\Phi$ 
under $\mathcal{T}_{\beta}$ is reported in Eq. (5). The KMS condition then becomes

$$
\langle O[\Phi]\rangle=\left\langle O\left[\mathcal{T}_{\beta} \Phi\right]\right\rangle_{\tilde{S}}
$$

In Sec. VI A, on the basis of Eq. (48), we derive the typical form of the FDR, which involves the correlation function of two classical fields and the susceptibility expressed as a correlation between one quantum and one classical field.

3. In the grand canonical ensemble with density matrix $\rho=e^{-\beta(H-\mu N)} / \operatorname{tr} e^{-\beta(H-\mu N)}$, where $N=\int_{\mathbf{x}} \psi^{\dagger}(\mathbf{x}) \psi(\mathbf{x})$ is the particle number operator, the KMS condition Eq. (43) has to be generalized. In order to derive it, we split again the density matrix into a product $e^{-\beta(H-\mu N) / 2} \times e^{-\beta(H-\mu N) / 2}$ (cf. the caption of Fig. 1). Then, moving one of the two factors through each of the blocks of operators $A$ and $B$ as in the second (orange) box in panel (b) of Fig. 1 has not only the effect of adding $+i \beta / 2$ and $-i \beta / 2$ to the time arguments of the field operators in $A$ and $B$ respectively, as in the case $\mu=0$; taking into account the canonical commutation relations, additional factors appears due to the fact that

$$
\begin{aligned}
e^{\sigma \beta \mu N / 2} \psi(\mathbf{x}) e^{-\sigma \beta \mu N / 2} & =e^{-\sigma \beta \mu / 2} \psi(\mathbf{x}), \\
e^{\sigma \beta \mu N / 2} \psi^{\dagger}(\mathbf{x}) e^{-\sigma \beta \mu N / 2} & =e^{\sigma \beta \mu / 2} \psi^{\dagger}(\mathbf{x}),
\end{aligned}
$$

where $\sigma=+1$ and -1 for operators which are part of the time-ordered and anti-time-ordered blocks of operators $A$ and $B$, respectively. The factors $e^{ \pm \sigma \beta \mu / 2}$ can be taken out of the expectation value in Eq. (43) (corresponding to the trace in Fig. $1(b)$ ) and do not affect the restoration of time order by means of the time-reversal transformation, which is illustrated in the last (green) box in panel (b) of Fig. 1. Therefore, on the r.h.s. of Eq. (47) they would appear as prefactors, which are absorbed in the modified transformation given in Eq. (6).

\section{From the KMS condition to a symmetry of the Schwinger-Keldysh action}

In the previous section we showed that the KMS condition within the Schwinger-Keldysh functional integral formalism takes the form of Eq. (47) (with $\mathcal{T}_{\beta}$ given by either Eq. (3) or (6)). Here we argue further, that the latter relation is equivalent to requiring the invariance of the Schwinger-Keldysh action under the equilibrium symmetry $\mathcal{T}_{\beta}$. To this end, we express the expectation values on the left and right hand sides of Eq. (47) as the functional integrals

$$
\langle O[\Psi]\rangle=\int \mathcal{D}[\Psi] O[\Psi] e^{i S[\Psi]}
$$

and

$$
\left\langle O\left[\mathcal{T}_{\beta} \Psi\right]\right\rangle_{\tilde{S}}=\int \mathcal{D}[\Psi] O\left[\mathcal{T}_{\beta} \Psi\right] e^{i \tilde{S}[\Psi]},
$$

respectively. Performing a change of integration variables $\Psi \rightarrow \mathcal{T}_{\beta} \Psi$ in the last expression, the argument of $O$ simplifies because $\mathcal{T}_{\beta} \Psi \rightarrow \mathcal{T}_{\beta}^{2} \Psi=\Psi$, since $\mathcal{T}_{\beta}$ is involutive (see Sec. III). In addition, we show in Appendix D that the absolute value of the determinant of the Jacobian $\mathcal{J}=\delta\left(\mathcal{T}_{\beta} \Psi\right) / \delta \Psi$ associated with $\mathcal{T}_{\beta}$ equals one, i.e., $|\operatorname{Det} \mathcal{J}|=1$, and therefore the integration measure is not affected by the change of variable. Accordingly, one has

$$
\left\langle O\left[\mathcal{T}_{\beta} \Psi\right]\right\rangle_{\tilde{S}}=\int \mathcal{D}[\Psi] O[\Psi] e^{i \tilde{S}\left[\mathcal{T}_{\beta} \Psi\right]},
$$

and by comparing this expression to Eq. (50) a sufficient condition for their equality is indeed Eq. (7), which expresses the invariance of the Schwinger-Keldysh action under the equilibrium transformation. Since the observable $O[\Psi]$ in Eqs. (47), (50), and (52) is arbitrary, the condition is also necessary, which proves that Eqs. (7) and (47) (and, consequently, the KMS condition) are equivalent.

\section{EXAMPLES}

In this section we discuss some concrete examples of how the invariance of a certain Schwinger-Keldysh action under the equilibrium transformation $\mathcal{T}_{\beta}$ can be used in practice. First we show that Eq. (47) (or, equivalently, Eq. (48)) contains as a special case the quantum FDR which establishes a relationship between the two-time correlation function of the field $\phi_{c}$ (see Eq. (4)) and its response to an external perturbation which couples linearly to it. This was also noted in Ref. 40; however, the conceptual advance done here consists in realizing that the FDR can be regarded as a WardTakahashi identity associated with the equilibrium symmetry. In Sec. VI B, instead, we elaborate on the non-equilibrium nature of Markovian dynamics described by a quantum master equation (of the Lindblad form), which is seen to violate explicitly the equilibrium symmetry.

The case of a system which is driven out of equilibrium by a coupling to different baths is considered in Sec. VIC. In particular, we discuss a single bosonic mode coupled to two baths at different temperatures and chemical potentials. Finally, in Sec. VID we briefly list a number of additional applications of the symmetry. Some of those have been put into practice in the context of classical dynamical systems, and could be generalized to the quantum case with the aid of the symmetry transformation discussed in the present paper.

\section{A. Fluctuation-dissipation relation for two-time functions}

Considering the invariance of the Schwinger-Keldysh action under $\mathcal{T}_{\beta}$ in Eq. (5) as the defining property of thermodynamic equilibrium, the generalized FDR in Eq. (48) (which, as discussed above, is nothing but the Ward-Takahashi identity associated with the symmetry), emerges as a consequence of equilibrium conditions. Then, from the generalized FDR in Eq. (48), the FDR for two-time functions $[55,56]$ can indeed 
be derived as a special case. The latter reads

$$
\begin{aligned}
G^{K}(\omega, \mathbf{q}) & =\left(G^{R}(\omega, \mathbf{q})-G^{A}(\omega, \mathbf{q})\right) \operatorname{coth}(\beta \omega / 2) \\
& =i 2 \operatorname{Im} G^{R}(\omega, \mathbf{q}) \operatorname{coth}(\beta \omega / 2),
\end{aligned}
$$

where the Keldysh, retarded, and advanced Green's functions $G^{K}, G^{R}$, and $G^{A}$, respectively, are related to expectation values of classical and quantum fields via

$$
\begin{aligned}
& i G^{K}(\omega, \mathbf{q})(2 \pi)^{d+1} \delta\left(\omega-\omega^{\prime}\right) \delta^{(d)}\left(\mathbf{q}-\mathbf{q}^{\prime}\right)=\left\langle\phi_{c}(\omega, \mathbf{q}) \phi_{c}^{*}\left(\omega^{\prime}, \mathbf{q}^{\prime}\right)\right\rangle, \\
& i G^{R}(\omega, \mathbf{q})(2 \pi)^{d+1} \delta\left(\omega-\omega^{\prime}\right) \delta^{(d)}\left(\mathbf{q}-\mathbf{q}^{\prime}\right)=\left\langle\phi_{c}(\omega, \mathbf{q}) \phi_{q}^{*}\left(\omega^{\prime}, \mathbf{q}^{\prime}\right)\right\rangle, \\
& i G^{A}(\omega, \mathbf{q})(2 \pi)^{d+1} \delta\left(\omega-\omega^{\prime}\right) \delta^{(d)}\left(\mathbf{q}-\mathbf{q}^{\prime}\right)=\left\langle\phi_{q}(\omega, \mathbf{q}) \phi_{c}^{*}\left(\omega^{\prime}, \mathbf{q}^{\prime}\right)\right\rangle .
\end{aligned}
$$

Here we are assuming translational invariance in both time and space, which is reflected in the appearance of frequencyand momentum-conserving $\delta$-functions in the previous expressions. The FDR valid in classical systems [48, 49] can be recovered from the quantum FDR Eq. (53) by taking the classical limit as described in Sec. IV C. Contrary to what one might suspect at a first glance from the appearance of the time-reversed action $\tilde{S}$ in the generalized FDR in Eq. (48), the derivation of the FDR for two-time functions we present below is valid irrespective of whether the action contains external fields which break TRI or not.

Let us consider the identity Eq. (48) for specific choices of the functional $O[\Phi]$. In particular, by taking $O$ to be equal to $\phi_{q} \phi_{q}^{*}$, the expectation value on the 1.h.s. of Eq. (48) has to vanish due to causality [55] and therefore

$$
0=\left\langle\phi_{q}(\omega, \mathbf{q}) \phi_{q}^{*}\left(\omega^{\prime}, \mathbf{q}^{\prime}\right)\right\rangle=\left\langle\mathcal{T}_{\beta} \phi_{q}(\omega,-\mathbf{q}) \mathcal{T}_{\beta} \phi_{q}^{*}\left(\omega^{\prime},-\mathbf{q}^{\prime}\right)\right\rangle_{\tilde{S}}
$$

Upon inserting the expression of the fields transformed according to Eq. (5), one readily finds the FDR

$$
G_{\tilde{S}}^{K}(\omega, \mathbf{q})=\left(G_{\tilde{S}}^{R}(\omega, \mathbf{q})-G_{\tilde{S}}^{A}(\omega, \mathbf{q})\right) \operatorname{coth}(\beta \omega / 2)
$$

with the time-reversed action $\tilde{S}$. By setting, instead, $O$ equal to the product of two classical fields, the 1.h.s. of Eq. (48) renders the Keldysh Green's function $G^{K}$ in Eq. (54); by using the explicit form of $\mathcal{T}_{\beta}$ in Eq. (5) and the FDR derived above, the r.h.s. of that equation coincides with $G_{\tilde{S}}^{K}$ and therefore one concludes that

$$
G_{\tilde{S}}^{K}(\omega, \mathbf{q})=G^{K}(\omega,-\mathbf{q}),
$$

which expresses the transformation behavior of the Keldysh Green's function under time reversal of the Hamiltonian. Finally, by replacing $O[\Phi]$ in Eq. (48) with the product $\phi_{c}(\omega, \mathbf{q}) \phi_{q}^{*}\left(\omega^{\prime}, \mathbf{q}\right)$ of one classical and one quantum field, the 1.h.s. renders by definition the retarded Green's function $G^{R}$ while the r.h.s. can be worked out as explained above. Taking into account Eq. (56), one can eliminate the Keldysh Green's function $G^{K}$ appearing on the r.h.s. in favor of the retarded and advanced Green's functions $G^{R}$ and $G^{A}$, respectively, and eventually finds

$$
G_{\tilde{S}}^{R}(\omega, \mathbf{q})=G^{R}(\omega,-\mathbf{q})
$$

This relation, together with its complex conjugate (which relates the advanced Green's functions calculated from the original and time-reversed Hamiltonians, respectively) and Eqs. (57) and (56), yields the FDR (53).

\section{B. Non-equilibrium nature of steady states of quantum master equations}

In classical statistical physics, the coupling of a system to a thermal bath and the resulting relaxation to thermodynamic equilibrium are commonly modelled in terms of Markovian stochastic processes, which can be described, e.g., by suitable Langevin equations with Gaussian white noise [76]. The Markovian dynamics of a quantum system, on the other hand, is described by a quantum master equation in the Lindblad form $[59,60]$ (or by an equivalent Schwinger-Keldysh functional integral). Under specific conditions on the structure of the Lindblad operator [85, 94], the stationary state of this dynamics is described by a thermal Gibbs distribution, such that all static properties (equal-time correlation functions) are indistinguishable from those in thermodynamic equilibrium. In spite of this fact, however, dynamical signatures of thermodynamic equilibrium such as the KMS condition (see Sec. V C) and the FDR (see Sec. VI A) are violated [95, 96]. This violation can be traced back to the fact that the Markovian and rotating wave approximations, which are done in deriving this quantum master equation cause an explicit breaking of the equilibrium symmetry as we show in this section - i.e., although the system is coupled to a bath in thermodynamic equilibrium, the system itself does not reach equilibrium. Physically, this can be understood by noting that the microscopic dynamics underlying an approximate Markovian behavior in this case is indeed driven. A typical example in the context of quantum optics is an atom with two relevant energy levels separated by a level spacing $\omega_{0}$ and subject to an external driving laser with frequency $v$ detuned from resonance by an amount $\Delta=v-\omega_{0} \ll \omega_{0}$. Transitions between the ground and the excited state are made possible only by the driving laser, and the energy scale which controls the validity of the Markov approximation in the dynamics of the two-level system is set by $\omega_{0}$. The excited state is assumed to be unstable and it can undergo spontaneous decay by emitting a photon to the radiation field, which acts as a reservoir. This illustrates the combined driven and dissipative nature of such quantum optical systems.

In order to investigate in more detail the effect of the Markovian approximation typically done in the driven context on the validity of the equilibrium symmetry $\mathcal{T}_{\beta}$, we consider a system with a certain action $S$, whose degrees of freedom are linearly coupled to those of a thermal bath. By integrating out the latter degrees of freedom, a dissipative contribution to the original action is generated. In order to simplify the discussion, we assume that the bath consists of non-interacting harmonic oscillators $b_{\mu, \sigma}(t)$, labelled by an index $\mu$ (with $\sigma$ referring to the branch of the Schwinger-Keldysh contour), with proper frequency $\omega_{\mu}$, which are in thermodynamic equilibrium at a temperature $T=1 / \beta$. The Schwinger-Keldysh 
action of the bath is then given by

$$
\begin{aligned}
S_{b}=\sum_{\mu} \int_{t, t^{\prime}}( & \left.b_{\mu,+}^{*}(t), b_{\mu,-}^{*}(t)\right) \\
& \times\left(\begin{array}{ll}
G_{\mu}^{++}\left(t, t^{\prime}\right) & G_{\mu}^{+-}\left(t, t^{\prime}\right) \\
G_{\mu}^{-+}\left(t, t^{\prime}\right) & G_{\mu}^{--}\left(t, t^{\prime}\right)
\end{array}\right)^{-1}\left(\begin{array}{l}
b_{\mu,+}\left(t^{\prime}\right) \\
b_{\mu,-}\left(t^{\prime}\right)
\end{array}\right)
\end{aligned}
$$

where the Green's functions $i G_{\mu}^{\sigma \sigma^{\prime}}\left(t, t^{\prime}\right)=\left\langle b_{\mu, \sigma}(t) b_{\mu, \sigma^{\prime}}^{*}\left(t^{\prime}\right)\right\rangle$ for the oscillators of the bath are fixed by requiring it to be in equilibrium and therefore they read $[55,56]$

$$
\begin{aligned}
& G_{\mu}^{+-}\left(t, t^{\prime}\right)=-i n\left(\omega_{\mu}\right) e^{-i \omega_{\mu}\left(t-t^{\prime}\right)}, \\
& G_{\mu}^{-+}\left(t, t^{\prime}\right)=-i\left(n\left(\omega_{\mu}\right)+1\right) e^{-i \omega_{\mu}\left(t-t^{\prime}\right)}, \\
& G_{\mu}^{++}\left(t, t^{\prime}\right)=\theta\left(t-t^{\prime}\right) G_{\mu}^{-+}\left(t, t^{\prime}\right)+\theta\left(t^{\prime}-t\right) G_{\mu}^{+-}\left(t, t^{\prime}\right), \\
& G_{\mu}^{--}\left(t, t^{\prime}\right)=\theta\left(t^{\prime}-t\right) G_{\mu}^{-+}\left(t, t^{\prime}\right)+\theta\left(t-t^{\prime}\right) G_{\mu}^{+-}\left(t, t^{\prime}\right) .
\end{aligned}
$$

Here $n(\omega)=1 /\left(e^{\beta \omega}-1\right)$ is the Bose distribution function and $\theta(t)$ denotes the Heaviside step function, which is defined as

$$
\theta(t)= \begin{cases}1, & t \geq 0 \\ 0, & t<0 .\end{cases}
$$

The coupling $S_{s b}$ between the system and the bath is assumed to be linear in the bath variables and have a strength $\sqrt{\gamma_{\mu}}$,

$$
\begin{aligned}
S_{s b}=\sum_{\mu} \sqrt{\gamma_{\mu}} \int_{t}\left(L_{+}^{*}(t) b_{\mu,+}(t)+L_{+}(t) b_{\mu,+}^{*}(t)\right. \\
\left.-L_{-}^{*}(t) b_{\mu,-}(t)-L_{-}(t) b_{\mu,-}^{*}(t)\right) .
\end{aligned}
$$

Here, $L_{ \pm}(t)$ are associated with the quantum jump or Lindblad operators, which we assume to be quasilocal polynomials of the system's bosonic fields $\left\{\psi_{ \pm}, \psi_{ \pm}^{*}\right\}$ resulting from normally ordered operators in a second quantized description (e.g., the simplest choice would be $\left.L_{ \pm}(t)=\psi_{ \pm}(t, \mathbf{x})\right)$. In order to simplify the notation, we do not indicate here the spatial dependence of the fields (both of the system and of the bath), which is understood together with the corresponding integration over space. We assume the harmonic oscillators which constitute the bath to be spatially uncorrelated. The Schwinger-Keldysh functional integral with total action $S+S_{b}+S_{s b}$ involving both system and bath degrees of freedom is quadratic in the latter and, therefore, the bath can be integrated out. The resulting contribution is

$$
\begin{aligned}
S^{\prime}=-\int_{\omega_{0}-\vartheta}^{\omega_{0}+\vartheta} \mathrm{d} \omega & \gamma(\omega) v(\omega) \int_{t, t^{\prime}}\left(L_{+}^{*}(t),-L_{-}^{*}(t)\right) \\
& \times\left(\begin{array}{ll}
G_{\omega}^{++}\left(t, t^{\prime}\right) & G_{\omega}^{+-}\left(t, t^{\prime}\right) \\
G_{\omega}^{-+}\left(t, t^{\prime}\right) & G_{\omega}^{--}\left(t, t^{\prime}\right)
\end{array}\right)\left(\begin{array}{c}
L_{+}\left(t^{\prime}\right) \\
-L_{-}\left(t^{\prime}\right)
\end{array}\right),
\end{aligned}
$$

which eventually sums to $S$. In deriving this action, we made the additional assumption that the bath modes $\left\{\omega_{\mu}\right\}_{\mu}$ form a dense continuum with a spectral density $v(\omega)=\sum_{\mu} \delta\left(\omega-\omega_{\mu}\right)$, centered around a frequency $\omega_{0}$, with a bandwidth $\vartheta$ (see further below for its interpretation). Then, sums of the form $\sum_{\mu} \gamma_{\mu} \cdots$ can be approximated as integrals over frequencies $\int_{\omega_{0}-\vartheta}^{\omega_{0}+\vartheta} \mathrm{d} \omega \gamma(\omega) v(\omega) \cdots$, where $\gamma(\omega)$ describes the frequency distribution of the oscillator strengths. Inserting the explicit expressions (60) for the bath Green's functions into Eq. (63), we obtain

$$
\begin{aligned}
S^{\prime}=-i \int_{\omega_{0}-\vartheta}^{\omega_{0}+\vartheta} \frac{\mathrm{d} \omega}{2 \pi} \gamma(\omega) v(\omega)\left(n(\omega) L_{+}^{*}(\omega) L_{-}(\omega)+(n(\omega)+1) L_{-}^{*}(\omega) L_{+}(\omega)-\int_{-\infty}^{\infty} \frac{\mathrm{d} \omega^{\prime}}{2 \pi}\left\{\left[\theta\left(\omega^{\prime}-\omega\right)(n(\omega)+1)\right.\right.\right. \\
\left.\left.\left.+\theta\left(-\omega^{\prime}+\omega\right) n(\omega)\right] L_{+}^{*}\left(\omega^{\prime}\right) L_{+}\left(\omega^{\prime}\right)+\left[\theta\left(-\omega^{\prime}+\omega\right)(n(\omega)+1)+\theta\left(\omega^{\prime}-\omega\right) n(\omega)\right] L_{-}^{*}\left(\omega^{\prime}\right) L_{-}\left(\omega^{\prime}\right)\right\}\right),
\end{aligned}
$$

where $L_{\sigma}(\omega)$ describe the quantum jump operators in the frequency space, and $\theta(\omega)=i \mathcal{P} \frac{1}{\omega}+\pi \delta(\omega)$ (where $\mathcal{P}$ denotes the Cauchy principal value) is the Fourier transform of $\theta(t)$ in Eq. (61). The terms involving the principal value contribute to the Hamiltonian part of the total Schwinger-Keldysh action of the system $S+S^{\prime}$. Assuming that the jump operators are quasilocal polynomials of the bosonic field operators of the system, $L_{\sigma}(\omega)$ transform under $\mathcal{T}_{\beta^{\prime}}$ as the field operators, i.e.,

$$
\mathcal{T}_{\beta^{\prime}} L_{\sigma}(\omega)=e^{-\sigma \beta^{\prime} \omega / 2} L_{\sigma}^{*}(\omega) \text { and } \mathcal{T}_{\beta^{\prime}} L_{\sigma}^{*}(\omega)=e^{\sigma \beta^{\prime} \omega / 2} L_{\sigma}(\omega) ;
$$

inserting these expressions in Eq. (64) one finds that the contour-diagonal terms (i.e., those proportional to $L_{\sigma}^{*}(\omega) L_{\sigma}(\omega)$ with $\sigma= \pm 1$, which include, in particular, the above-mentioned principal value terms) are invariant due to frequency conservation (cf. the discussion in Sec. IV A 2). On 
the other hand, for the contribution which is off-diagonal in the Schwinger-Keldysh contour one finds

$$
\begin{aligned}
& S_{\text {off-diag }}^{\prime}\left[\mathcal{T}_{\beta^{\prime}} \Psi\right]=-i \int_{\omega_{0}-\vartheta}^{\omega_{0}+\vartheta} \frac{\mathrm{d} \omega}{2 \pi} \gamma(\omega) v(\omega) \\
& \times\left[n(\omega) e^{\beta^{\prime} \omega} L_{+}(\omega) L_{-}^{*}(\omega)+(n(\omega)+1) e^{-\beta^{\prime} \omega} L_{-}(\omega) L_{+}^{*}(\omega)\right]
\end{aligned}
$$

If the value of $\beta^{\prime}$ matches the inverse temperature $\beta=1 / T$ of the bath modes, encoded in $n(\omega)$, it is easy to see that these terms are invariant under $\mathcal{T}_{\beta}$ because $n(\omega) e^{\beta \omega}=n(\omega)+1$. In summary, one concludes that $S^{\prime}\left[\mathcal{T}_{\beta} \Psi\right]=S^{\prime}[\Psi]$ and being also the action $S$ of the system in isolation invariant under $\mathcal{T}_{\beta}$, the same holds for the total effective action $S+S^{\prime}$ of the system in contact with the thermal bath.

In order to understand the effect of the Markovian approximation in the driven context on the invariance under $\mathcal{T}_{\beta}$, let us now consider Eq. (64) after this approximation has been done. In particular, in order for these approximations to be valid, we assume that one can choose a "rotating" frame in which the evolution of the system is slow compared to the energy scales $\omega_{0}$ and $\vartheta$ which characterize the bath. This is possible if the system is driven by an external classical field such as a laser, so that the frequency of the drive bridges the gap between the natural time scales of the system and those of the bath. Then, all jump operators in Eq. (63) may be evaluated at the same time $t$, since the integral kernel in Eq. (63) (i.e., the product of bath Green's functions $G_{\omega}^{\sigma \sigma^{\prime}}$, density of states $v(\omega)$ and oscillator strength distribution $\gamma(\omega)$, integrated over the bath bandwidth) differs from zero only within a correlation time $\tau_{c} \approx 1 / \vartheta$, which is assumed to be much shorter than the timescale over which $L_{\sigma}(t)$ evolves in the rotating frame. Additionally we assume that the spectral density $v(\omega)$ of the states of the bath and the corresponding coupling strength $\sqrt{\gamma(\omega)}$ to the system do not vary appreciably within the relevant window $\omega_{0}-\vartheta<\omega<\omega_{0}+\vartheta$, such that one can set $\gamma(\omega) v(\omega) \approx \gamma\left(\omega_{0}\right) v\left(\omega_{0}\right)$. As before, the terms in Eq. (64) which are diagonal in the contour indices are invariant un$\operatorname{der} \mathcal{T}_{\beta}$ (see Eq. (65)) also after the Markovian approximation; accordingly, we focus on the off-diagonal terms in Eq. (66), which become

$$
\begin{aligned}
& S_{\text {off-diag }}^{\prime}[\Psi]=-i \gamma\left(\omega_{0}\right) v\left(\omega_{0}\right) \\
& \quad \times \int_{-\infty}^{\infty} \frac{\mathrm{d} \omega}{2 \pi}\left[\bar{n} L_{+}^{*}(\omega) L_{-}(\omega)+(\bar{n}+1) L_{-}^{*}(\omega) L_{+}(\omega)\right],
\end{aligned}
$$

where $\bar{n}=n\left(\omega_{0}\right)$ is the occupation number of the bath modes at frequency $\omega_{0}$. This makes it clear that $\vartheta$ acts as a highfrequency cutoff, whose precise value, under Markovian conditions, does not affect the physics. Applying the transformation $\mathcal{T}_{\beta}$ to the fields one has

$$
\begin{aligned}
& S_{\text {off-diag }}^{\prime}\left[\mathcal{T}_{\beta} \Psi\right]=-i \gamma\left(\omega_{0}\right) v\left(\omega_{0}\right) \\
& \times \int_{-\infty}^{\infty} \frac{\mathrm{d} \omega}{2 \pi}\left[\bar{n} e^{\beta \omega} L_{+}(\omega) L_{-}^{*}(\omega)+(\bar{n}+1) e^{-\beta \omega} L_{-}(\omega) L_{+}^{*}(\omega)\right]
\end{aligned}
$$

In order for $S_{\text {off-diag }}^{\prime}$ to be invariant under $\mathcal{T}_{\beta}$, this expression should be equal to $S_{\text {off-diag }}^{\prime}[\Psi]$ in Eq. (67), which requires $\bar{n} e^{\beta \omega}=\bar{n}+1$ for all values of the frequencies $\omega$ within the relevant region $\omega_{0}-\vartheta<\omega<\omega_{0}+\vartheta$. Clearly, this is not possible and therefore the equilibrium symmetry is explicitly broken by the Markovian approximation in the driven context.

\section{System coupled to different baths}

A simple way to drive a system out of equilibrium is to bring it in contact with baths at different temperatures and chemical potentials. In this case, a net flux of energy and particles is established across the system, preventing it from thermalizing and, consequently, causing a violation of the symmetry $\mathcal{T}_{\beta}$. This scenario occurs, e.g., in the context of quantum electronics in a quantum dot connected by tunnel electrodes to two leads, between which a finite voltage difference is maintained. Here, for simplicity, we consider a minimal bosonic counterpart of this system, constituted by a single bosonic mode - described by the fields $\left\{\psi_{ \pm}, \psi_{ \pm}^{*}\right\}$ coupled to two baths of non-interacting harmonic oscillators kept at different temperatures and chemical potentials. We show explicitly that the non-equilibrium nature of this setup is accompanied by a violation of the equilibrium symmetry. The generalization of this argument to a multi-mode system or to a larger number of baths is straightforward.

We consider two baths of non-interacting harmonic oscillators $b_{i, v}$, where the label $i=1,2$ denotes the bath to which the operator belongs, while $v$ denotes the corresponding mode with frequency $\omega_{i, v}$. Each bath is assumed to be in thermodynamic equilibrium with different inverse temperatures $\beta_{1}$, $\beta_{2}$ and different chemical potentials $\mu_{1}, \mu_{2}$. The SchwingerKeldysh action $S_{b, i}$ of each bath takes the same form as in Eq. (59). However, in the present case, the distribution functions $n_{i}(\omega)$ entering the bath Green's functions depend on the chemical potentials $\mu_{i}$ as $n_{i}(\omega)=1 /\left(e^{\beta_{i}\left(\omega-\mu_{i}\right)}-1\right)$. As in the previous section, the bath is assumed to be coupled linearly to the system variables $L_{ \pm}(t)$ which are quasilocal polynomials of the bosonic fields $\left\{\psi_{ \pm}, \psi_{ \pm}^{*}\right\}$. The dynamics of the system and the baths is then controlled by a functional integral with the total Schwinger-Keldysh action $S+S_{b, 1}+S_{b, 2}+S_{s b}$, where $S$ is to the action of the system, i.e., the single bosonic mode, and $S_{s b}$ is the system-bath coupling. As in the previous section, an effective dynamics for the system's variables can be obtained by integrating out those of the bath. This yields an effective action 


$$
\begin{array}{r}
S^{\prime}=-i \int_{-\infty}^{+\infty} \frac{\mathrm{d} \omega}{2 \pi} \tilde{J}(\omega)\left(\tilde{n}(\omega) L_{+}^{*}(\omega) L_{-}(\omega)+(\tilde{n}(\omega)+1) L_{-}^{*}(\omega) L_{+}(\omega)-\int_{-\infty}^{\infty} \frac{\mathrm{d} \omega^{\prime}}{2 \pi}\left\{\left[\theta\left(\omega^{\prime}-\omega\right)(\tilde{n}(\omega)+1)\right.\right.\right. \\
\left.\left.\left.+\theta\left(-\omega^{\prime}+\omega\right) \tilde{n}(\omega)\right] L_{+}^{*}\left(\omega^{\prime}\right) L_{+}\left(\omega^{\prime}\right)+\left[\theta\left(-\omega^{\prime}+\omega\right)(\tilde{n}(\omega)+1)+\theta\left(\omega^{\prime}-\omega\right) \tilde{n}(\omega)\right] L_{-}^{*}\left(\omega^{\prime}\right) L_{-}\left(\omega^{\prime}\right)\right\}\right) .
\end{array}
$$

The action $S^{\prime}$ is formally similar to the one in Eq. (64), with the spectral density $\gamma(\omega) v(\omega)$ replaced by the sum of the spectral densities of the baths $\tilde{J}(\omega)=J_{1}(\omega)+J_{2}(\omega)$, where $J_{i}(\omega)=\gamma_{i}(\omega) v_{i}(\omega)$ (with $v_{i}(\omega)$ and $\gamma_{i}(\omega)$ defined as in Sec. VIB, see after Eq. (63)). Analogously, the distribution function $n(\omega)$ of the single bath we considered in Sec. VIB is replaced by the average of the distribution functions of the two baths $i=1,2$ weighted by the relative spectral densities, i.e.,

$$
\tilde{n}(\omega)=\frac{J_{1}(\omega)}{J_{1}(\omega)+J_{2}(\omega)} n_{1}(\omega)+\frac{J_{2}(\omega)}{J_{1}(\omega)+J_{2}(\omega)} n_{2}(\omega)
$$

Now, we consider how the effective action (69) transforms under the thermal symmetry $\mathcal{T}_{\beta}$. Since here we are explicitly considering the presence of chemical potentials, we will use the generalization of the symmetry $\mathcal{T}_{\beta, \mu}$ in Eq. (6). As discussed in Sec. VIB, since $L_{ \pm}, L_{ \pm}^{*}$ are quasilocal polynomials of the bosonic fields of the system, they transform under $\mathcal{T}_{\beta, \mu}$ as

$$
\begin{aligned}
& \mathcal{T}_{\beta, \mu} L_{\sigma}(\omega)=e^{-\sigma \beta(\omega-\mu) / 2} L_{\sigma}^{*}(\omega), \\
& \mathcal{T}_{\beta, \mu} L_{\sigma}^{*}(\omega)=e^{\sigma \beta(\omega-\mu) / 2} L_{\sigma}(\omega) .
\end{aligned}
$$

Accordingly, the products $L_{\sigma}(\omega) L_{\sigma}^{*}(\omega)$ are invariant under the symmetry and therefore the contour-diagonal part of $S^{\prime}$, which contains such terms, is invariant. On the other hand, the part $S_{\text {off-diag }}^{\prime}$ of $S^{\prime}$ which is off-diagonal in the SchwingerKeldysh contour (i.e., the first two terms on the r.h.s. of Eq. (69)) is modified as:

$$
\begin{aligned}
S_{\text {off-diag }}^{\prime}\left[\mathcal{T}_{\beta, \mu} \Psi\right]= & -i \int_{-\infty}^{+\infty} \frac{\mathrm{d} \omega}{2 \pi} \tilde{J}(\omega) \\
\times & {\left[\tilde{n}(\omega) e^{\beta(\omega-\mu)} L_{+}(\omega) L_{-}^{*}(\omega)\right.} \\
& \left.+(\tilde{n}(\omega)+1) e^{-\beta(\omega-\mu)} L_{-}(\omega) L_{+}^{*}(\omega)\right]
\end{aligned}
$$

Comparing this expression with Eq. (69), one readily sees that the invariance of this term under $\mathcal{T}_{\beta, \mu}$ requires $\tilde{n}(\omega) e^{\beta(\omega-\mu)}=$ $\tilde{n}(\omega)+1$, and therefore $S_{\text {off-diag }}^{\prime}$ is not invariant under $\mathcal{T}_{\beta, \mu}$, unless the two baths have the same temperature and chemical potential, i.e., $\beta_{1}=\beta_{2}=\beta$ and $\mu_{1}=\mu_{2}=\mu$. In this case, one can easily verify from Eq. (70) that the average distribution function $\tilde{n}(\omega)$ is just the Bose-Einstein distribution $\tilde{n}(\omega)=1 /\left(e^{\beta(\omega-\mu)}-1\right)$ and, as a consequence, $\tilde{n}(\omega) e^{\beta(\omega-\mu)}=$ $\tilde{n}(\omega)+1$.

In conclusion, when the system is driven out of equilibrium by a net flux of energy or particles, induced by a difference between the temperatures or the chemical potentials of the baths, the total action of the system is no longer invariant under $\mathcal{T}_{\beta, \mu}$, as $S^{\prime}\left[\mathcal{T}_{\beta, \mu} \Psi\right] \neq S^{\prime}[\Psi]$.

\section{Further applications}

Among the various possible applications of the symmetry $\mathcal{T}_{\beta}$, we mention here:

a. Symmetry-preserving approximations. Properties of interacting many-body systems can usually be obtained only by resorting to certain approximations. Then, while FDRs for correlation and response functions can be established exactly in the absence of interactions, in an approximate inclusion of the latter one has to make sure that the FDRs are not broken. In other words, the approximation should conserve the thermal symmetry. This requirement for classical statistical systems has been implemented in the mode-coupling theory of the glass transition in Ref. 97.

The field-theoretic formalism provides the natural framework for studying the behavior of systems at long wavelengths and low energies by employing renormalization-group methods. In any of these methods, an effective description which is obtained by integrating out fast fluctuations must have the same symmetries as those present at microscopic scales. For example, in the case of the functional renormalization group (for reviews see Refs. 98-102), this is achieved by choosing an ansatz to approximate the scale-dependent effective action which incorporates these symmetries. In this context, the classical limit of the thermal symmetry discussed here has been used in functional renormalization group studies of model A [103] and model C [104]. The quantum thermal symmetry, instead, is analogously preserved by the ansatz for the effective action chosen in Ref. [105] (in the form of FDT), where the scale-dependent crossover from quantum to classical dynamics is studied. Alternatively, one can devise approximation schemes which are compatible with the equivalent KMS conditions, as discussed in detail in Ref. 40. Note, however, that in this work the KMS condition in the form of Eq. (47) is imposed on the scale-dependent Green's functions (supplemented by the corresponding condition on the vertex functions). On the other hand, the symmetry constraint can directly be applied to the effective action itself, which is the generating functional of vertex functions and contains information on all correlation functions.

b. Fluctuation relations. Another concrete example of the usefulness of the thermal symmetry is provided by the derivation of transient fluctuation relations [106, 107] for time-dependent particle transport in Ref. [58]. There, the symmetry is generalized in order to account for the presence of a time-dependent counting field which probes the current flowing through the system. This generalized symmetry yields a relation analogous to Eq. (47) (however, formulated in terms 
of the generating functional for correlation and response functions), from which, e.g., a fluctuation relation for the probability distribution of work done on the system and the transmitted charge can be derived.

\section{CONCLUSIONS}

We demonstrated here that the Schwinger-Keldysh action describing the dynamics of a generic quantum many-body system acquires a certain symmetry $\mathcal{T}_{\beta}$ if the evolution occurs in thermal equilibrium. To a certain extent, this symmetry was discussed in Ref. 58 in the specific context of fluctuation relations for particle transport. We traced the origin of this symmetry back to the Kubo-Martin-Schwinger (KMS) condition which establishes a relationship between multi-time correlation functions in real and imaginary times of a system in canonical equilibrium at a certain temperature. Fluctuationdissipation relations are then derived as the Ward-Takahashi identities associated with $\mathcal{T}_{\beta}$. Remarkably, in the classical limit, this equilibrium symmetry reduces to the one known in classical stochastic systems, where it was derived from the assumption of detailed balance. By comparing with this classical case, important questions on the nature of equilibrium in quantum systems arise. In particular, while microreversibility and detailed balance of the dynamics are deeply connected to the notion of equilibrium in classical stochastic systems, an analogous relationship for quantum systems does not clearly emerge and surely deserves further investigation.

The equilibrium symmetry $\mathcal{T}_{\beta}$ is expected to play a crucial role in the study of thermalization in quantum systems, in particular when combined with a renormalization-group analysis. In fact, on the one hand, it provides a simple but powerful theoretical tool to assess whether a certain system is able to reproduce thermal equilibrium. This can indeed be accomplished by a direct inspection of the microscopic Schwinger-Keldysh action (or of the effective one generated after integrating out some degrees of freedom, e.g., along a renormalization-group flow) which describes the dynamics of the system, rather than checking, for instance, the validity of the fluctuation-dissipation relations among various correlation functions. On the other hand, the equilibrium symmetry might be useful also in order to investigate or characterize possible departures from equilibrium and, in this respect, it would be interesting to consider the case in which the system evolves in a generalized Gibbs ensemble [6, 8, 65-71]. Finally, while we focussed here on the case of bosons, the extension of our analysis to different statistics, for instance fermionic and spin systems, represents an interesting issue.

Acknowledgements. - We would like to thank A. Altland, J. Berges, I. Carusotto, L. F. Cugliandolo, T. Gasenzer, J. M. Pawlowski, T. Prosen, H. Spohn, C. Wetterich, and especially A. Rosch for useful discussions. L. M. S. and S. D. gratefully acknowledge support by the START Grant No. Y 581-N16. S. D. also thanks the German Research Foundation for support via ZUK 64.

\section{Appendix A: Invariance of quadratic dissipative contributions}

In order to show explicitly the invariance of $S_{d}$ in Eq. (19) under the transformation $\mathcal{T}_{\beta}$ in Eq. (5), it is convenient to assume that $h(\omega, \mathbf{q})$ as a function of the frequency $\omega$ has definite parity and to consider separately the cases of odd and even functions, $h_{o}(-\omega, \mathbf{q})=-h_{o}(\omega, \mathbf{q})$ and $h_{e}(-\omega, \mathbf{q})=h_{e}(\omega, \mathbf{q})$, respectively. The generic case follows straightforwardly by linear combination. We then consider how the actions

$$
\begin{aligned}
S_{d}=i \epsilon \int_{\omega, \mathbf{q}} \Phi_{q}^{\dagger} & (\omega, \mathbf{q})\left\{\begin{array}{c}
h_{o}(\omega, \mathbf{q}) \\
h_{e}(\omega, \mathbf{q}) \sigma_{z}
\end{array}\right\} \\
& \times\left(\Phi_{c}(\omega, \mathbf{q})+\operatorname{coth}(\beta \omega / 2) \Phi_{q}(\omega, \mathbf{q})\right)
\end{aligned}
$$

— where $\Phi$ is introduced in Sec. IV A 1, see Eq. (12) — with a certain $\beta$ transform under a transformation $\mathcal{T}_{\beta^{\prime}}$ [see Eq. (5)] of the fields, with a generic parameter $\beta^{\prime}$. One finds

$$
\begin{aligned}
& S_{d}\left[\mathcal{T}_{\beta^{\prime}} \Phi\right]=i \epsilon \int_{\omega, \mathbf{q}}\left(\sinh \left(\beta^{\prime} \omega / 2\right) \Phi_{c}^{\dagger}(-\omega, \mathbf{q})+\cosh \left(\beta^{\prime} \omega / 2\right) \Phi_{q}^{\dagger}(-\omega, \mathbf{q})\right) \sigma_{x}\left\{\begin{array}{c}
h_{o}(\omega, \mathbf{q}) \\
h_{e}(\omega, \mathbf{q}) \sigma_{z}
\end{array}\right\} \sigma_{x} \\
& \quad \times\left[\cosh \left(\beta^{\prime} \omega / 2\right) \Phi_{c}(-\omega, \mathbf{q})-\sinh \left(\beta^{\prime} \omega / 2\right) \Phi_{q}(-\omega, \mathbf{q}) \operatorname{coth}(\beta \omega / 2)\left(-\sinh \left(\beta^{\prime} \omega / 2\right) \Phi_{c}(-\omega, \mathbf{q})+\cosh \left(\beta^{\prime} \omega / 2\right) \Phi_{q}(-\omega, \mathbf{q})\right)\right] .
\end{aligned}
$$

Note that the terms involving solely the classical field spinor $\Phi_{c}$ cancel each other only if $\beta^{\prime}=\beta$. Otherwise, terms $\propto \Phi_{c}^{\dagger} \Phi_{c}$ remain, which lead to a violation of causality [55]. For $\beta^{\prime}=\beta$ instead, we obtain

$$
\begin{aligned}
S_{d}\left[\mathcal{T}_{\beta} \Phi\right]=-i \epsilon \int_{\omega, \mathbf{q}}\left(-\sinh (\beta \omega / 2) \Phi_{c}^{\dagger}(\omega, \mathbf{q})+\cosh (\beta \omega / 2) \Phi_{q}^{\dagger}(\omega, \mathbf{q})\right) & \left\{\begin{array}{c}
h_{o}(\omega, \mathbf{q}) \\
h_{e}(\omega, \mathbf{q}) \sigma_{z}
\end{array}\right\} \\
& \times(\sinh (\beta \omega / 2)-\operatorname{coth}(\beta \omega / 2) \cosh (\beta \omega / 2)) \Phi_{q}(\omega, \mathbf{q}) .
\end{aligned}
$$

By means of the identity

$$
\sinh x-\operatorname{coth} x \cosh x=-1 / \sinh x,
$$

and after some straightforward algebraic manipulations one eventually finds that $S_{d}\left[\mathcal{T}_{\beta} \Phi\right]=S_{d}[\Phi]$, i.e., that $S_{d}$ (with a certain $\beta$ ) is invariant under $\mathcal{T}_{\beta}$. 


\section{Appendix B: Invariance of dissipative vertices}

As pointed out in the main text, a first constraint that has to be imposed on the functions $f_{1,2,3}$ appearing in the dissipative vertex in Eq. (20) follows from the requirement of causality of the Schwinger-Keldysh action. The latter must vanish when $\psi_{+}=\psi_{-}$[55], which implies the condition

$$
\begin{gathered}
\int_{\omega_{1}, \ldots, \omega_{4}} \delta\left(\omega_{1}-\omega_{2}+\omega_{3}-\omega_{4}\right) \psi_{+}^{*}\left(\omega_{1}\right) \psi_{+}\left(\omega_{2}\right) \psi_{+}^{*}\left(\omega_{3}\right) \psi_{+}\left(\omega_{4}\right) \\
\times\left[f_{1}\left(\omega_{1}, \omega_{2}, \omega_{3}, \omega_{4}\right)+f_{2}\left(\omega_{1}, \omega_{2}, \omega_{3}, \omega_{4}\right)\right. \\
\left.+f_{3}\left(\omega_{1}, \omega_{2}, \omega_{3}, \omega_{4}\right)\right]=0 .
\end{gathered}
$$

Now let us consider Eq. (20) with the transformed fields $\mathcal{T}_{\beta} \Psi_{\sigma}$, i.e., $S_{d}\left[\mathcal{T}_{\beta} \Psi\right]$. Requiring it to be equal to $S_{d}[\Psi]$, we find that the following conditions should be fulfilled:

$$
\begin{aligned}
& f_{1}\left(\omega_{1}, \omega_{2}, \omega_{3}, \omega_{4}\right)-f_{1}\left(\omega_{2}, \omega_{1}, \omega_{4}, \omega_{3}\right)=0, \\
& f_{2}\left(\omega_{1}, \omega_{2}, \omega_{3}, \omega_{4}\right)-f_{2}\left(\omega_{2}, \omega_{1}, \omega_{4}, \omega_{3}\right)=0, \\
& f_{3}\left(\omega_{1}, \omega_{2}, \omega_{3}, \omega_{4}\right)-e^{\beta\left(\omega_{1}-\omega_{2}\right)} f_{3}\left(\omega_{2}, \omega_{1}, \omega_{4}, \omega_{3}\right)=0,
\end{aligned}
$$

where we used the conservation of frequencies implied by the $\delta$-function in Eq. (20) to simplify the exponent in the last line. Specifically, the necessary conditions are that the expressions on the 1.h.s. of these relations should vanish when integrated over frequencies after having been multiplied by the corresponding combinations of fields in Eq. (20) and by the $\delta$-function on frequencies. The relations in Eq. (B2) are, however, sufficient conditions for the equality of $S_{d}\left[\mathcal{T}_{\beta} \Psi\right]$ and $S_{d}[\Psi]$.

To begin with, we investigate the possible existence of a frequency-independent solution of Eqs. (B1) and (B2) for $f_{1,2,3}$; these two equations then imply

$$
f_{1}=-f_{2}=\text { constant } \text { and } f_{3}=0 \text {. }
$$

However, this solution can be seen to lack physical relevance for the following reason: any physically sensible dissipative contribution to the Schwinger-Keldysh action compatible with the thermal symmetry can be considered as originating from integrating out a thermal bath which is appropriately coupled to the system. Anticipating the discussion of Sec. VIB, we note that such dissipative contributions always involve terms which are not diagonal in the contour indices (cf. Eq. (63)). $S_{d}$ with $f_{1,2,3}$ given by Eq. (B3), however, is not of this form. In fact, inserting Eq. (B3) in Eq. (20) yields a vertex that is equal to the two-body interaction in Eq. (11) apart from an overall factor of $i$, i.e., such a vertex would originate from an imaginary two-body coupling in a Hamiltonian. Clearly, this would violate hermiticity, rendering the Hamiltonian unphysical.

While this demonstrates that - as anticipated in the main text - a frequency-independent number-conserving quartic vertex is not compatible with equilibrium conditions, solutions of Eqs. (B1) and (B2) do exist with $f_{i}$ depending on frequency. One particular solution is given by Eq. (21) of the main text.

\section{Appendix C: Representation of correlation functions in the Schwinger-Keldysh formalism}

c. Two-time correlation functions. In order to derive the representation of a two-time correlation function in the Schwinger-Keldysh formalism reported in Eq. (31), we insert the explicit expressions (30) for the Heisenberg operators $A\left(t_{A}\right)$ and $B\left(t_{B}\right)$ in the trace which defines the 1.h.s. of Eq. (31) according to Eq. (29). Then, by introducing an additional and arbitrary time $t_{f}$ such that $t_{i}<t_{A, B}<t_{f}$, and by using the cyclic property of the trace one can write

$$
\left\langle A\left(t_{A}\right) B\left(t_{B}\right)\right\rangle=\operatorname{tr}\left(e^{-i H\left(t_{f}-t_{B}\right)} B e^{-i H\left(t_{B}-t_{i}\right)} \rho e^{i H\left(t_{A}-t_{i}\right)} A e^{i H\left(t_{f}-t_{A}\right)}\right) .
$$

The evolution of the density matrix is adjoint to the evolution of Heisenberg operators, i.e., $\rho(t)=e^{-i H t} \rho e^{i H t}$. Thus, the operator $e^{-i H\left(t-t^{\prime}\right)}\left(e^{i H\left(t-t^{\prime}\right)}\right)$ acting from the left (right) on the density matrix $\rho$ corresponds to the evolution in time from $t^{\prime}$ to $t$. In the correlation function $(\mathrm{C} 1)$, the time evolution from $t_{i}$ to $t_{f}$ on the left (right) of $\rho$ is intercepted by the operator $B$ at time $t_{B}\left(A\right.$ at time $\left.t_{A}\right)$. In order to convert the r.h.s. of Eq. (C1) into a path integral, the standard procedure (see, e.g., Refs. 55 and 56) to be followed consists in writing the exponentials of the evolution operators as infinite products of infinitesimal and subsequent temporal evolutions (Trotter decomposition), in-between of which one can introduce completeness relations in terms of coherent states carrying the additional label "+" on the left of the density matrix, and a "-" on its right. These coherent states are eventually labeled by a temporal index on the forward (+) and backward (-) branches of the close-time path which characterizes the resulting action. Correspondingly, the operators on the left and on the right of the density matrix ( $B$ and $A$, respectively, in Eq. (C1)) turn out to be evaluated on the fields (i.e., coherent states) which are defined, respectively, on the forward and backward branches of the closed time path and this yields immediately the equality in Eq. (31), where the ordering of the matrix elements $A_{-}$and $B_{+}$on its r.h.s. is inconsequential. For the sake of completeness, we note that the expression as a Schwinger-Keldysh functional integral of a two-time function is not unique: in fact, it is straightforward to check that, by rearranging operators in Eq. (C1), one can equivalently arrive at

$$
\left\langle A\left(t_{A}\right) B\left(t_{B}\right)\right\rangle= \begin{cases}\left\langle A_{+}\left(t_{A}\right) B_{+}\left(t_{B}\right)\right\rangle & \text { for } t_{A}>t_{B}, \\ \left\langle A_{-}\left(t_{A}\right) B_{-}\left(t_{B}\right)\right\rangle & \text { for } t_{A}<t_{B} .\end{cases}
$$

However, as discussed below, the choice of Eq. (31) naturally lends itself to a generalization to multi-time correlation functions.

d. Multi-time correlation functions. The functional integral on the r.h.s. of Eq. (33) relation can be constructed from a straightforward generalization of Eq. (C1): after a reshuffling of the operators such that $A$ and $B$ appear respectively on the left and right of the density matrix — as explained above the temporal evolution can be artificially extended from $t_{i}$ to $t_{f}$ and it is intercepted on the 1.h.s. of the density matrix by operators $b_{1}, \ldots, b_{M}$ at times $t_{B, 1}, \ldots, t_{B, M}$ and on the r.h.s. by operators $a_{1}, \ldots, a_{N}$ at times $t_{A, 1}, \ldots, t_{A, N}$. Again, the resulting expression for the correlation function can be converted 
directly into a path integral by a Trotter decomposition of the subsequent evolutions and by inserting completeness relations in terms of coherent states carrying the label "+" corresponding to the forward contour on the 1.h.s. of the density matrix and the label "-" for the backward contour on the r.h.s., which eventually leads to Eq. (33).

\section{Appendix D: Jacobian of the equilibrium transformation}

In order to prove that $|\operatorname{Det} \mathcal{J}|=1$ it is convenient to calculate the Jacobian $\mathcal{J}$ associated with Eq. (3) in frequency and momentum space, which reads

$$
\mathcal{J}\left(\omega, \mathbf{q}, \omega^{\prime}, \mathbf{q}^{\prime}\right)=(2 \pi)^{d} \delta^{(d)}\left(\mathbf{q}+\mathbf{q}^{\prime}\right) J\left(\omega, \omega^{\prime}\right)
$$

where

$$
J\left(\omega, \omega^{\prime}\right)=2 \pi \delta\left(\omega-\omega^{\prime}\right)\left(\begin{array}{cccc}
0 & e^{\beta \omega / 2} & 0 & 0 \\
e^{-\beta \omega / 2} & 0 & 0 & 0 \\
0 & 0 & 0 & e^{\beta \omega / 2} \\
0 & 0 & e^{-\beta \omega / 2} & 0
\end{array}\right)
$$

The eigenvectors $v_{i}$ and eigenvalues $\lambda_{i}$ of the frequencydependent part, i.e., the solutions of the equation

$$
\int \frac{\mathrm{d} \omega^{\prime}}{2 \pi} J\left(\omega, \omega^{\prime}\right) v_{i}\left(\omega^{\prime}\right)=\lambda_{i} v_{i}(\omega)
$$

are

$$
\begin{array}{ll}
v_{1}(\omega)=\left(0,0,-e^{\beta \omega / 2}, 1\right)^{T}, & v_{2}(\omega)=\left(-e^{\beta \omega / 2}, 1,0,0\right)^{T}, \\
v_{3}(\omega)=\left(0,0, e^{\beta \omega / 2}, 1\right)^{T}, & v_{4}(\omega)=\left(e^{-\beta \omega / 2}, 1,0,0\right)^{T},
\end{array}
$$

with $\lambda_{1}=\lambda_{2}=-1$, and $\lambda_{3}=\lambda_{4}=1$, so that Det $J=$ $\lambda_{1} \lambda_{2} \lambda_{3} \lambda_{4}=1$. As for the momentum-dependent part of the Jacobian matrix Eq. (D1), we note that its eigenvectors can be constructed with any function $f(\mathbf{q})$ by taking the even and odd combinations $f(\mathbf{q}) \pm f(-\mathbf{q})$ :

$$
\int_{\mathbf{q}^{\prime}}(2 \pi)^{d} \delta^{(d)}\left(\mathbf{q}+\mathbf{q}^{\prime}\right)\left(f\left(\mathbf{q}^{\prime}\right) \pm f\left(-\mathbf{q}^{\prime}\right)\right)= \pm(f(\mathbf{q}) \pm f(-\mathbf{q})) \text {. }
$$

Thus the eigenvalues of this part are \pm 1 , and hence the absolute value of the Jacobian matrix is $|\operatorname{Det} \mathcal{J}|=1$.
[1] A. Polkovnikov, K. Sengupta, A. Silva, and M. Vengalattore, Rev. Mod. Phys. 83, 863 (2011).

[2] V. Yukalov, Laser Phys. Lett. 8, 485 (2011).

[3] J. Eisert, M. Friesdorf, and C. Gogolin, Nat. Phys. 11, 124 (2015).

[4] A. Lamacraft and J. Moore, in Ultracold Bosonic and Fermionic Gases, edited by A. Fetter, K. Levin, and D. Stamper-Kurn (Elsevier, Oxford, 2012) Chap. 7.

[5] I. Bloch, J. Dalibard, and W. Zwerger, Rev. Mod. Phys. 80, 885 (2008).

[6] E. T. Jaynes, Phys. Rev. 106, 620 (1957).

[7] T. Kinoshita, T. Wenger, and D. S. Weiss, Nature 440, 900 (2006).

[8] M. Rigol, V. Dunjko, V. Yurovsky, and M. Olshanii, Phys. Rev. Lett. 98, 050405 (2007).

[9] M. Kollar, F. Wolf, and M. Eckstein, Phys. Rev. B 84, 054304 (2011).

[10] J.-S. Caux and R. Konik, Phys. Rev. Lett. 109, 175301 (2012).

[11] D. Basko, I. Aleiner, and B. Altshuler, Ann. Phys. (N. Y). 321, 1126 (2006).

[12] A. Pal and D. A. Huse, Phys. Rev. B 82, 174411 (2010).

[13] M. Serbyn, Z. Papić, and D. Abanin, Phys. Rev. Lett. 111, 127201 (2013).

[14] R. Vosk and E. Altman, Phys. Rev. Lett. 110, 067204 (2013).

[15] D. Rossini, A. Silva, G. Mussardo, and G. E. Santoro, Phys. Rev. Lett. 102, 127204 (2009).

[16] A. Mitra and T. Giamarchi, Phys. Rev. Lett. 107, 150602 (2011).

[17] L. Foini, L. F. Cugliandolo, and A. Gambassi, Phys. Rev. B 84, 212404 (2011).

[18] L. Foini, L. F. Cugliandolo, and A. Gambassi, J. Stat. Mech. Theory Exp. 2012, P09011 (2012).

[19] I. Carusotto and C. Ciuti, Rev. Mod. Phys. 85, 299 (2013).

[20] T. Byrnes, N. Y. Kim, and Y. Yamamoto, Nat. Phys. 10, 803 (2014).
[21] M. Hartmann, F. Brandão, and M. Plenio, Laser Photonics Rev. 2, 527 (2008).

[22] A. A. Houck, H. E. Türeci, and J. Koch, Nat. Phys. 8, 292 (2012).

[23] R. Blatt and C. F. Roos, Nat. Phys. 8, 277 (2012).

[24] F. Marquardt and S. M. Girvin, Physics (College. Park. Md). 2, 40 (2009).

[25] A. Mitra, S. Takei, Y. B. Kim, and A. J. Millis, Phys. Rev. Lett. 97, 236808 (2006).

[26] S. Diehl, A. Micheli, A. Kantian, B. Kraus, H. P. Büchler, and P. Zoller, Nat. Phys. 4, 878 (2008).

[27] S. Diehl, A. Tomadin, A. Micheli, R. Fazio, and P. Zoller, Phys. Rev. Lett. 105, 015702 (2010).

[28] E. G. Dalla Torre, E. Demler, T. Giamarchi, and E. Altman, Nat. Phys. 6, 806 (2010).

[29] E. G. Dalla Torre, E. Demler, T. Giamarchi, and E. Altman, Phys. Rev. B 85, 184302 (2012).

[30] E. G. D. Dalla Torre, S. Diehl, M. D. Lukin, S. Sachdev, and P. Strack, Phys. Rev. A 87, 023831 (2013).

[31] A. Chiocchetta and I. Carusotto, Europhys. Lett. 102, 67007 (2013).

[32] L. M. Sieberer, S. D. Huber, E. Altman, and S. Diehl, Phys. Rev. Lett. 110, 195301 (2013).

[33] U. C. Täuber and S. Diehl, Phys. Rev. X 4, 021010 (2014).

[34] M. Wouters and I. Carusotto, Phys. Rev. B 74, 245316 (2006).

[35] A. Mitra and T. Giamarchi, Phys. Rev. B 85, 075117 (2012).

[36] B. Öztop, M. Bordyuh, O. E. Müstecaplolu, and H. E. Türeci, New J. Phys. 14, 085011 (2012).

[37] M. F. Maghrebi and A. V. Gorshkov, arXiv:1507.01939 .

[38] K.-c. Chou, Z.-b. Su, B.-1. Hao, and L. Yu, Phys. Rep. 118, 1 (1985).

[39] E. Wang and U. Heinz, Phys. Rev. D 66, 025008 (2002).

[40] S. G. Jakobs, M. Pletyukhov, and H. Schoeller, J. Phys. A Math. Theor. 43, 103001 (2010). 
[41] A. Messiah, Quantum Mechanics II, 3rd ed. (North-Holland Pubsishing Company, Amsterdam, 1965).

[42] R. Kubo, J. Phys. Soc. Japan 12, 570 (1957).

[43] P. Martin and J. Schwinger, Phys. Rev. 115, 1342 (1959).

[44] H. Janssen, Z. Phys. B Condens. Matter 23, 377 (1976).

[45] R. Bausch, H. K. Janssen, and H. Wagner, Z. Phys. B Condens. Matter 24, 113 (1976).

[46] H. Janssen, in Dynamical Critical Phenomena and Related Topics, Lecture Notes in Physics, Vol. 104, edited by C. Enz (Springer, Berlin, Heidelberg, 1979).

[47] H. Janssen, in From Phase Transitions to Chaos, Topics in Modern Statistical Physics, edited by G. Györgyi, I. Kondor, L. Sasvári, and T. Tél (World Scientific, Singapore, 1992).

[48] C. Aron, G. Biroli, and L. F. Cugliandolo, J. Stat. Mech. 2010, P11018 (2010).

[49] C. Aron, D. G. Barci, L. F. Cugliandolo, Z. G. Arenas, and G. S. Lozano, arXiv:1412.7564 .

[50] J. Schwinger, J. Math. Phys. 2, 407 (1961).

[51] P. M. Bakshi and K. T. Mahanthappa, J. Math. Phys. 4, 1 (1963).

[52] P. M. Bakshi and K. T. Mahanthappa, J. Math. Phys. 4, 12 (1963).

[53] K. Mahanthappa, Phys. Rev. 126, 329 (1962).

[54] L. V. Keldysh, Sov. Phys. JETP 20, 1018 (1965).

[55] A. Kamenev, Field Theory of Non-Equilibrium Systems (Cambridge University Press, Cambridge, 2011).

[56] A. Altland and B. Simons, Condensed Matter Field Theory, 2nd ed. (Cambridge University Press, Cambridge, 2010).

[57] H. T. C. Stoof, J. Low Temp. Phys. 114, 11 (1999).

[58] A. Altland, A. De Martino, R. Egger, and B. Narozhny, Phys. Rev. B 82, 115323 (2010).

[59] A. Kossakowski, Reports Math. Phys. 3, 247 (1972).

[60] G. Lindblad, Commun. Math. Phys. 48, 119 (1976).

[61] E. M. Lifshitz and L. P. Pitaevskii, Statistical Physics, Part 2: Theory of the Condensed State, 2nd ed. (Pergamon Press, New York, 1980).

[62] In Ref. 58, the symmetry is stated in terms of the real phase variables of complex fields. Then, the complex conjugation in Eq. (1) should be replaced by a change of sign.

[63] P. C. Hohenberg and B. I. Halperin, Rev. Mod. Phys. 49, 435 (1977).

[64] R. Folk and G. Moser, J. Phys. A. Math. Gen. 39, R207 (2006).

[65] A. Iucci and M. A. Cazalilla, Phys. Rev. A 80, 063619 (2009).

[66] T. Barthel and U. Schollwöck, Phys. Rev. Lett. 100, 100601 (2008).

[67] G. Goldstein and N. Andrei, arXiv:1405.4224 .

[68] B. Pozsgay, M. Mestyán, M. Werner, M. Kormos, G. Zaránd, and G. Takács, Phys. Rev. Lett. 113, 117203 (2014).

[69] M. Mierzejewski, P. Prelovšek, and T. Prosen, Phys. Rev. Lett. 113, 020602 (2014)

[70] B. Wouters, J. De Nardis, M. Brockmann, D. Fioretto, M. Rigol, and J.-S. Caux, Phys. Rev. Lett. 113, 117202 (2014).

[71] F. H. L. Essler, G. Mussardo, and M. Panfil, arXiv:1411.5352

[72] M. P. A. Fisher, G. Grinstein, and D. S. Fisher, Phys. Rev. B 40, 546 (1989).

[73] P. C. Martin, E. D. Siggia, and H. A. Rose, Phys. Rev. A 8, 423 (1973).

[74] De Dominicis, C., J. Phys. Colloques 37, C1 (1976).

[75] C. De Dominicis, Phys. Rev. B 18, 4913 (1978).

[76] U. C. Täuber, Critical Dynamics: A Field Theory Approach to Equilibrium and Non-Equilibrium Scaling Behavior (Cambridge University Press, Cambridge, 2014).
[77] L. M. Sieberer, S. D. Huber, E. Altman, and S. Diehl, Phys. Rev. B 89, 134310 (2014).

[78] C. Gardiner, Handbook of stochastic methods for physics, chemistry and the natural sciences, 3rd ed., Springer Series in Synergetics (Springer, Berlin, Heidelberg, 2004).

[79] J. Zinn-Justin, Quantum Field Theory and Critical Phenomena, 4th ed., International Series of Monographs on Physics No. 113 (Oxford University Press, Oxford, 2002).

[80] G. S. Agarwal, Z. Phys. 258, 409 (1973).

[81] R. Alicki, Reports Math. Phys. 10, 249 (1976).

[82] A. Kossakowski, A. Frigerio, V. Gorini, and M. Verri, Commun. Math. Phys. 57, 97 (1977).

[83] A. Frigerio and V. Gorini, Commun. Math. Phys. 93, 517 (1984).

[84] W. A. Majewski, J. Math. Phys. 25, 614 (1984).

[85] R. Alicki and K. Lendi, Quantum Dynamical Semigroups and Applications, Lecture Notes in Physics, Vol. 717 (Springer, Berlin, Heidelberg, 2007).

[86] H. J. Carmichael and D. F. Walls, Z. Phys. B Condens. Matter 23, 299 (1976).

[87] J. M. Deutsch, Phys. Rev. A 43, 2046 (1991).

[88] M. Srednicki, Phys. Rev. E 50, 888 (1994).

[89] M. Srednicki, J. Phys. A: Math. Gen. 32, 1163 (1999).

[90] E. Khatami, G. Pupillo, M. Srednicki, and M. Rigol, Phys. Rev. Lett. 111, 050403 (2013).

[91] Here we used the notion of "Ward-Takahashi identity" in the slightly generalized sense which encompasses the case of identities between correlation functions resulting from discrete symmetries (such as Eq. (3) in Sec. V D, which leads to, c.f., Eqs. (47) and (48)) beyond the usual case of continuous symmetries [79].

[92] We note that this condition is usually expressed in the form

$$
\left\langle A\left(t_{A}\right) B\left(t_{B}\right)\right\rangle=\left\langle B\left(t_{B}\right) A\left(t_{A}+i \beta\right)\right\rangle .
$$

However, an equilibrium state is also stationary and therefore both time arguments on the r.h.s. can be translated by $-i \beta / 2$ which leads immediately to Eq. (42). Here we are assuming that the analytic continuation of real-time correlation functions into the complex plane is possible and unambiguous.

[93] It is straightforward to verify that the transformation $T$ is not modified in the presence of complex time arguments.

[94] C. W. Gardiner and P. Zoller, Quantum Noise, 2nd ed., Springer series in synergetics, Vol. 56 (Springer, Berlin, Heidelberg, 2000).

[95] P. Talkner, Ann. Phys. (N. Y). 167, 390 (1986).

[96] G. W. Ford and R. F. O'Connell, Phys. Rev. Lett. 77, 798 (1996).

[97] A. Andreanov, G. Biroli, and A. Lefvre, J. Stat. Mech. Theory Exp. 2006, P07008 (2006).

[98] J. Berges, N. Tetradis, and C. Wetterich, Phys. Rept. 363, 223 (2002).

[99] J. M. Pawlowski, Ann. Phys. (N. Y). 322, 2831 (2007).

[100] B. Delamotte, in Renormalization Group and Effective Field Theory Approaches to Many-Body Systems SE - 2, Lecture Notes in Physics, Vol. 852, edited by A. Schwenk and J. Polonyi (Springer Berlin Heidelberg, 2012) pp. 49-132.

[101] O. J. Rosten, Phys. Rep. 511, 177 (2012).

[102] I. Boettcher, J. M. Pawlowski, and S. Diehl, Nucl. Phys. B Proc. Suppl. 228, 63 (2012).

[103] L. Canet and H. Chaté, J. Phys. A: Math. Theor. 40, 1937 (2007).

[104] D. Mesterházy, J. H. Stockemer, L. F. Palhares, and J. Berges, Phys. Rev. B 88, 174301 (2013). 
[105] D. Mesterházy, J. H. Stockemer, and Y. Tanizaki, arXiv:1504.07268 .

[106] M. Campisi, P. Hänggi, and P. Talkner, Rev. Mod. Phys. 83, 771 (2011).
[107] M. Esposito, U. Harbola, and S. Mukamel, Rev. Mod. Phys. 81, 1665 (2009). 